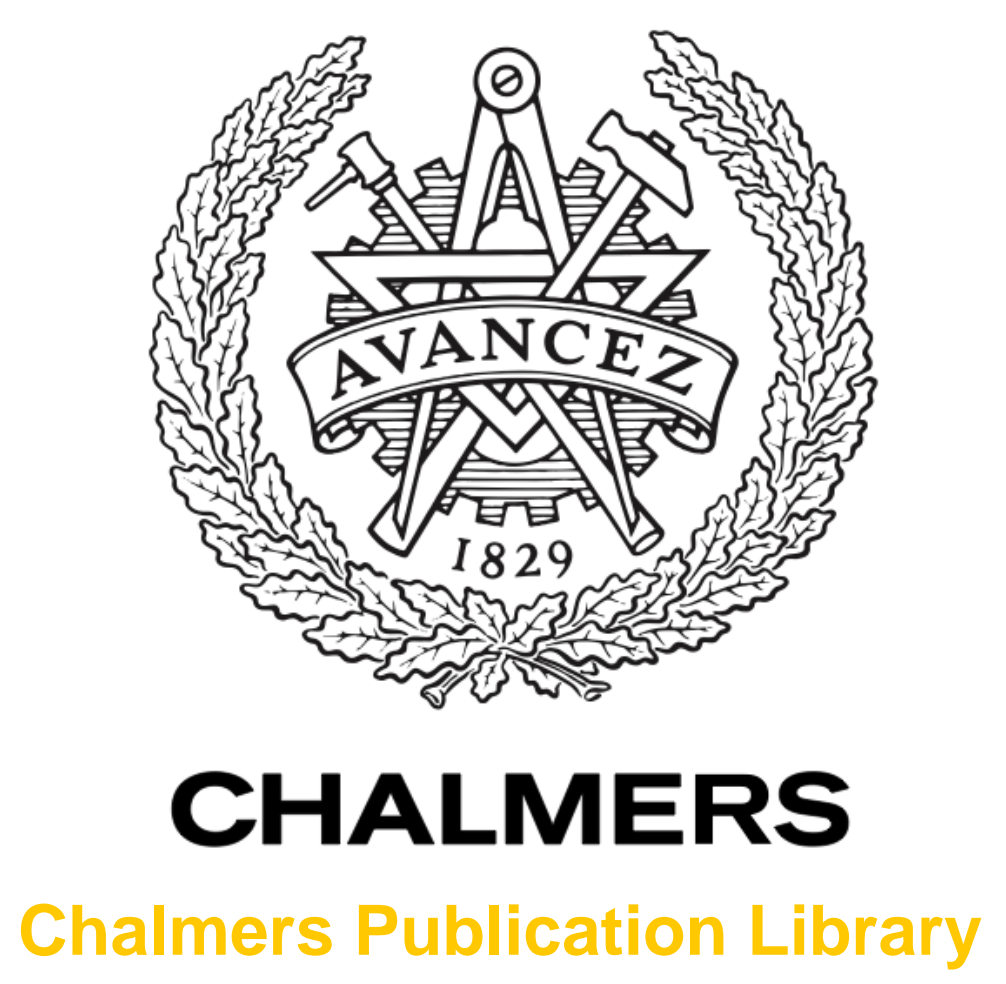

Self-Stabilizing TDMA Algorithms for Dynamic Wireless Ad Hoc Networks

This document has been downloaded from Chalmers Publication Library (CPL). It is the author's version of a work that was accepted for publication in:

International Journal of Distributed Sensor Networks (ISSN: 1550-1329)

Citation for the published paper:

Leone, P. ; Schiller, E. (2013) "Self-Stabilizing TDMA Algorithms for Dynamic Wireless Ad Hoc Networks". International Journal of Distributed Sensor Networks, vol. 2013(639761), pp. 17.

http://dx.doi.org/10.1155/2013/639761

Downloaded from: http://publications.lib.chalmers.se/publication/182509

Notice: Changes introduced as a result of publishing processes such as copy-editing and formatting may not be reflected in this document. For a definitive version of this work, please refer to the published source. Please note that access to the published version might require a subscription.

Chalmers Publication Library (CPL) offers the possibility of retrieving research publications produced at Chalmers University of Technology. It covers all types of publications: articles, dissertations, licentiate theses, masters theses, conference papers, reports etc. Since 2006 it is the official tool for Chalmers official publication statistics. To ensure that Chalmers research results are disseminated as widely as possible, an Open Access Policy has been adopted.

The CPL service is administrated and maintained by Chalmers Library. 


\title{
Research Article Self-Stabilizing TDMA Algorithms for Dynamic Wireless Ad Hoc Networks
}

\author{
Pierre Leone ${ }^{1}$ and Elad M. Schiller ${ }^{2}$ \\ ${ }^{1}$ Computer Science Department, Centre Universitaire d'Informatique, University of Geneva, Battelle bâtiment A, Route de Drize 7, \\ Carouge, 1227 Geneva, Switzerland \\ ${ }^{2}$ Chalmers University of Technology, Rännvägen 6B, 41296 Göteborg, Sweden
}

Correspondence should be addressed to Elad M. Schiller; elad@chalmers.se

Received 31 January 2013; Accepted 11 June 2013

Academic Editor: Kayhan Gulez

Copyright (C 2013 P. Leone and E. M. Schiller. This is an open access article distributed under the Creative Commons Attribution License, which permits unrestricted use, distribution, and reproduction in any medium, provided the original work is properly cited.

In dynamic wireless ad hoc networks (DynWANs), autonomous computing devices set up a network for the communication needs of the moment. These networks require the implementation of a medium access control (MAC) layer. We consider MAC protocols for DynWANs that need to be autonomous and robust as well as have high bandwidth utilization, high predictability degree of bandwidth allocation, and low communication delay in the presence of frequent topological changes to the communication network. Recent studies have shown that existing implementations cannot guarantee the necessary satisfaction of these timing requirements. We propose a self-stabilizing MAC algorithm for DynWANs that guarantees a short convergence period, and by that, it can facilitate the satisfaction of severe timing requirements, such as the above. Besides the contribution in the algorithmic front of research, we expect that our proposal can enable quicker adoption by practitioners and faster deployment of DynWANs that are subject changes in the network topology.

\section{Introduction}

Dynamic wireless ad hoc networks (DynWANs) are autonomous and self-organizing systems where computing devices require networking applications when a fixed network infrastructure is not available or not preferred to be used. In these cases, computing devices may set up a short-lived network for the communication needs of the moment, also known as an ad hoc network. Ad hoc networks are based on wireless communications that require implementation of a medium access control (MAC) layer. We consider MAC protocols for DynWANs that need to be autonomous and robust and have high bandwidth utilization, a high predictability degree of bandwidth allocation, and low communication delay [1] in the presence of frequent changes to the communication network topology. Existing implementations cannot guarantee the necessary satisfaction of timing requirements $[2,3]$. This work proposes an algorithmic design for self-stabilizing MAC protocols that guarantees a short convergence period and, by that, can facilitate the satisfaction of severe timing requirements. The proposed algorithm possesses a greater degree of predictability, while maintaining low communication delays and high throughput.

The dynamic and difficult-to-predict nature of wireless ad hoc networks give rise to many fault tolerance issues that requires efficient solutions. DynWANs, for example, are subject to transient faults due to hardware/software temporal malfunctions or short-lived violations of the assumed settings for modeling the location of the mobile nodes. Fault tolerant systems that are self-stabilizing [4] can recover after the occurrence of transient faults, which can cause an arbitrary corruption of the system state (so long as the program's code is still intact), or the model of dynamic networks in which communication links and nodes may fail and recover during normal operation [5]. The proof of self-stabilization requires convergence from an arbitrary starting system state. Moreover, once the system has converged and followed its specifications, it is required to do so forever. The selfstabilization design criteria liberate the application designer from dealing with low-level complications, such as bandwidth allocation in the presence of topology changes, and provide an important level of abstraction. Consequently, the 
application design can easily focus on its task and knowledgedriven aspects.

The IEEE 802.11 standard is widely used in wireless communications. Nonetheless, the research field of MAC protocols is very active and requires further investigation. In fact, the IEEE 802.11 amendment, IEEE 802.11p, for wireless access in vehicular environments (WAVE), has just being published. It was shown that the standard's existing implementations cannot guarantee channel access before a finite deadline $[2,3]$. Therefore, applications with severe timing requirements cannot predictably meet their deadlines, for example, safety-critical applications for vehicular systems.

ALOHAnet and its synchronized version slotted ALOHA [6] are pioneering wireless systems that employ a strategy of "random access." Time division multiple access (TDMA) [7] is another early approach, where nodes transmit one after the other, each using its own timeslot, say, according to a defined schedule. Radio transmission analysis in ad hoc networks [8] and relocation analysis of mobile nodes [9] show that there are scenarios in which MAC algorithms that employ a scheduled access strategy have lower throughput than algorithms that follow the random access strategy. However, the scheduled approach offers greater predictability of bandwidth allocation and communication delay, which can facilitate fairness [10] and energy conservation [11].

Our design choices have basic radio technology in mind, whilst aiming at satisfying applications that have severe timing requirements. We consider TDMA frames with fixed number of fixed-length timeslots. The design choice of TDMA frames with fixed-length radio time fits well applications that have severe delay requirements. By avoiding the division of fixed-length frames into timeslots of nonequal length, as in $[10,12]$, we take into consideration the specifications of basic radio technology.

In the context of the previous design choices, there are two well-known approaches for dealing with contention (timeslot exhaustion): (1) employing policies for administering message priority (for meeting timing requirements while maintaining high bandwidth utilization, such as [13]) or (2) adjusting the nodes' individual transmission signal strength or carrier sense threshold [14]. The former approach is widely accepted and adopted by the IEEE 802.11p standard, whereas the latter has only been evaluated via computer simulations. The proposed algorithm facilitates the implementation of both of the previous approaches. We consider implementation details of the standard approach in Section 7.

For the sake of presentation simplicity, we start by considering a single priority MAC protocol and base the timeslot allocation on vertex coloring, before considering multipriority implementation in Section 7. The proposed algorithm allocates timeslots to a number of nearby transmitters, that is, a number that is bounded by the TDMA frame size, whereas nonallocated transmitters receive busy channel indications. The analysis considers saturated situations in which the node degree in the message collision graph is smaller than the TDMA frame size. As explained previously, this analysis assumption does not restrict the number of concurrent transmitters when implementing the proposed MAC algorithm.

1.1. Related Work. We are not the first to propose a MAC algorithm for DynWANs that follows the TDMA's scheduled approach. STDMA [15] and Viqar and Welch [16] consider global navigation satellite system -based scheduling (GNSS) [17] according to the nodes' geographical position and their trajectories. Autonomous systems cannot depend on GNSS services, because they are not always available, or preferred not to be used, due to their cost. Arbitrarily long failure of signal loss can occur in underground parking lots and road tunnels. We propose a self-stabilizing TDMA algorithm that does not require GNSS accessibility or knowledge about the node trajectories. Rather, it considers an underlying selfstabilizing local pulse synchronization, such as [18, 19], which can be used for TDMA alignment; details appear in [18].

When using collision detection at the receiving side $[14,15,20-22]$, it is up to the receiving side to notify the sender about collisions via another round of collision-prone transmissions, say, by using frame information (FI) payload fields that include $T$ entries, where $T$ is the TDMA frame size. Thus far, the study of FI-based protocols has considered stochastic resolution of message collision via computer network simulation $[15,20,22-25]$. Simulations are also used for evaluating the heuristics of MS-ALOHA [14] for dealing with contention (timeslot exhaustion) by adjusting the nodes' individual transmission signal strength and/or carrier sense threshold. We do not consider lengthy frame information (FI) fields, which significantly increase the control information overhead, and yet we provide provable guarantee regarding the convergence time. Further analysis validation of the proposed algorithm via simulations and testbed implementation can be found in Section 8, and respectively, in [18].

The proposed algorithm does not consider collision detection mechanisms that are based on signal processing or hardware support, as in [26]. Rather, it employs a variation of a well-known strategy for eventually avoiding concurrent transmissions among neighbors. This strategy allows the sending side to eventually observe the existence of interfering transmissions. Before sending, the sender waits for a random duration while performing a clear channel assessment using basic radio technology (details appear in Section 3).

There are several MAC algorithms that are based on clear channel assessment. A recent example, [12], focuses on fair bandwidth allocation for single-hop-distance broadcasting while basing the interference model on discrete graphs. The authors do not consider self-stabilization. This work also considers clear channel assessment. However, we employ a strategy of random transmission delay in a way that allows the recipients to notice, in a probabilistic manner, prospective transmissions. We show that after a small number of rounds, the system is able to use the previous strategy for allocating the network bandwidth for single-hop-distance broadcasting when basing the interference model on discrete graphs. Further mitigation efforts of transmission pathologies, such as hidden terminal phenomena when unicast are considered, can be taken, for example, self-stabilizing two-hop-distance 
vertex coloring [27], equalizing transmission power, and coding-based methods [28], to name a few.

An abstract MAC layer was specified for DynWANs in [29]. The authors mention algorithms that can satisfy their specifications. However, they do not consider predictability.

Local algorithms [30,31] consider both theoretical and practical aspects of MAC algorithms ([32] and references therein) and the related problem of clock synchronization; see [33] and references therein. For example, the first partlyasynchronous self-organizing local algorithm for vertex coloring in wireless ad hoc networks is presented in [34]. However, this line currently does not consider dynamic networks and predictable bandwidth allocation.

Two examples of self-stabilizing TDMA algorithms are presented in $[10,35]$. The algorithms are based on vertexcoloring and the authors consider (nondynamic) ad hoc networks. Recomputation and floating output techniques ([4], Section 2.8) are used for converting deterministic local algorithms to self-stabilization in [36]. The authors focus on problems that are related to MAC algorithms. However, deterministic MAC algorithms are known to be inefficient in their bandwidth allocation when the topology of the communication network can change frequently [9]. There are several other proposals related to self-stabilizing MAC algorithms for sensor networks, for example, [37-40]; however, none of them consider dynamic networks, and their frame control information is quite extensive.

The MAC algorithms in $[9,18,41,42]$ have no proof that they are self-stabilizing. The authors of [9] present a MAC algorithm that uses convergence from a random starting state (inspired by self-stabilization). In [18, 41, 42], the authors use computer network simulators for evaluating self- $\star$ MAC algorithms.

1.2. Our Contribution. This work proposes a self-stabilizing MAC algorithm that demonstrates rapid convergence without the extensive use of frame control information. Our analysis shows that the algorithm facilitates the satisfaction of severe timing requirements for DynWANs.

We start by considering transient faults and topological changes to the communication network, that is, demonstrating self-stabilization in Theorem 2 . We then turn to focus on bounding the algorithm's convergence time after an arbitrary and unbounded finite sequence of transient faults and changes to the network topology. Theorem 3 shows that the expected local convergence time is brief and bounds it in (7). Theorem 7 formulates the expected global convergence time in (21). Moreover, for a given probability, the global convergence time is calculated in (22).

For discussion (Section 8), we point out the algorithm's ability to facilitate the satisfaction of severe timing requirements for DynWANs. Moreover, the analysis conclusions explain that when allowing merely a small fraction of the bandwidth to be spent on frame control information and when considering any given probability to converge within a bounded time, the proposed algorithm demonstrates a low dependency degree on the number of nodes in the network (as depicted by Figures 2 and 3).
We note that some of the proof details appear in the Appendix for the sake of presentation simplicity.

\section{Preliminaries}

The system consists of a set, $P$, of $N$ anonymous communicating entities, which we call nodes. Denote every node $p_{i} \in P$ with a unique index, $i$.

2.1. Synchronization. Each node has fine-grained, real-time clock hardware. We assume that the MAC protocol is invoked periodically by synchronous (common) pulse that aligns the starting time of the TDMA frame. This can be based, for example, on TDMA alignment algorithms [18], GPS [44], or a distributed pulse synchronization algorithm [19]. The term (broadcasting) timeslot refers to the period between two consecutive common pulses, $t_{x}$ and $t_{x+1}$, such that $t_{x+1}=$ $\left(t_{x} \bmod T\right)+1$, where $T$ is a predefined constant named the frame size. Throughout the paper, we assume that $T \geq$ 2. In our pseudocode, we use the event timeslot $(t)$ that is triggered by the common pulse. We assume that the timeslots are aligned as well.

2.2. Communications and Interferences. At any instance of time, the ability of any pair of nodes to communicate is defined by the set, $N_{i} \subseteq P$, of (direct) neighbors that node $p_{i} \in$ $P$ can communicate with directly. Wireless transmissions are subject to interferences (collisions). We consider the potential of the nodes to interfere with each other's communications. The interference model in this paper is based on discrete graphs.

The set $\mathcal{N}_{i} \supseteq N_{i}$ is the set of nodes that may interfere with $p_{i}$ 's communications when any nonempty subset of them, $I \subseteq \mathcal{N}_{i}: I \neq \emptyset$, transmits concurrently with $p_{i}$. We call $\mathcal{N}_{i}$ the (extended) neighborhood of node $p_{i} \in P$, and $d_{i}=\left|\mathcal{N}_{i}\right|$ is named the (extended) degree of node $p_{i}$. We assume that at any time, for any pair of nodes, $p_{i}, p_{j} \in P$; it holds that $p_{j} \in \mathcal{N}_{i}$ implies that $p_{i} \in \mathcal{N}_{j}$. Given a particular instance of time, we define the (interference) graph as $G:=(P, E)$, where $E:=\cup_{i \in P}\left\{\left(p_{i}, p_{j}\right): p_{j} \in \mathcal{N}_{i}\right\}$ represents the interference relationships among nodes.

2.3. Communication Schemes. We consider (basic technology of) radio units that raise the event carrier_sense() when they detect that the received energy levels have reached a threshold in which the radio unit is expected to succeed in carrier locking; see [45]. Timeslots allow the transmission of DATA packets using the primitives of transmit() and receive() after fetching (MAC_fetch()) a new packet from the upper layer, and respectively, before delivering (MAC_deliver()) the packet to the upper layer. A beacon is a short packet that includes no data load, rather the timing of the event carrier_sense() is the delivered information [12]. We assume that every node $p_{i} \in P$ that invokes the operation transmit() causes the event carrier_sense() to be raised by its neighbors, $p_{j} \in \mathcal{N}_{i}$, within the exposure time, $\varepsilon$. Before the transmission of the DATA packet in timeslot $t$, our communication scheme 


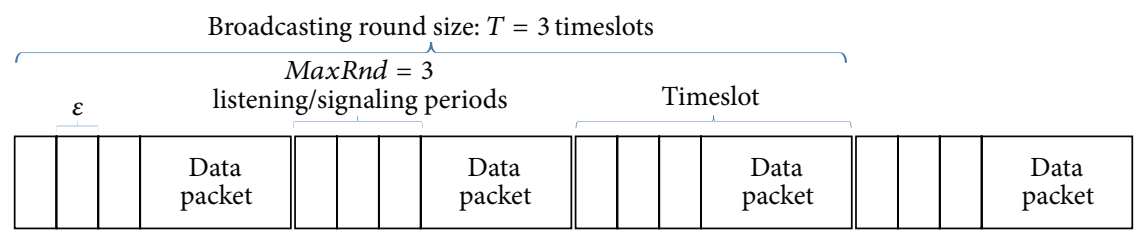

FIGURE 1: An example of TDMA frame, with three timeslots and three listening/signaling periods of size $\varepsilon$ (signal exposure time). Each timeslot has a constant number, MaxRnd $=4$, of listening/signaling periods in which beacons can be transmitted. The duration of each listening/signaling period is $\varepsilon$ (signal exposure time); the period during which a beacon that is sent by node $p_{i} \in P$ is transmitted and raises the ca received by all neighbors $p_{j} \in \mathcal{N}_{i}$. Namely, the period between $p_{i}$ 's transmission and $p_{j}$ 's rise of the carrier_sense() event.

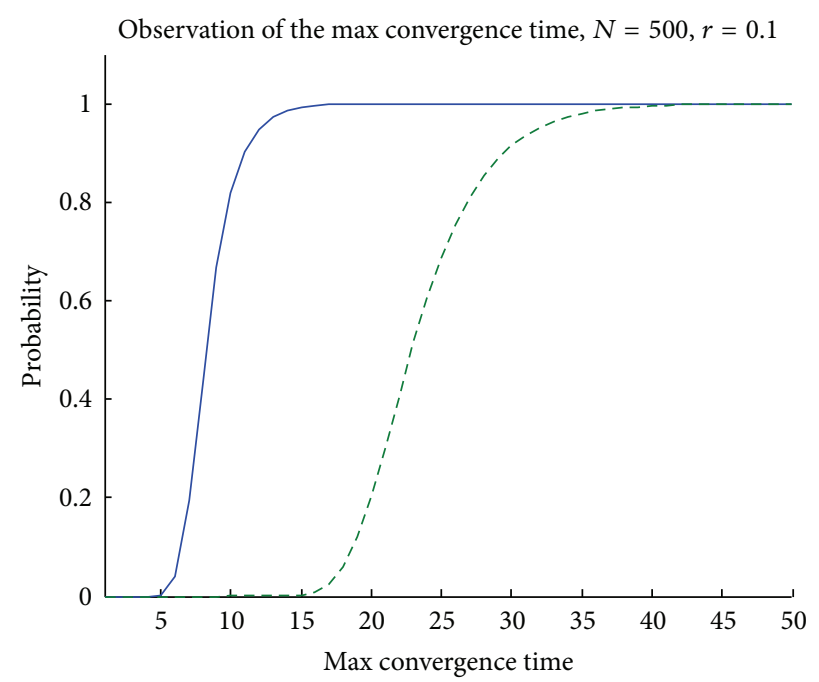

(a)

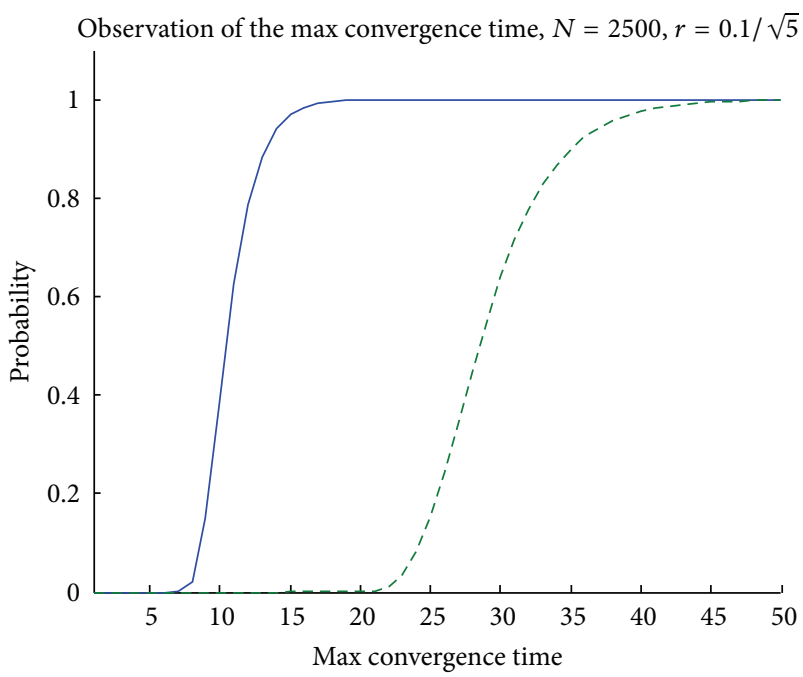

(b)

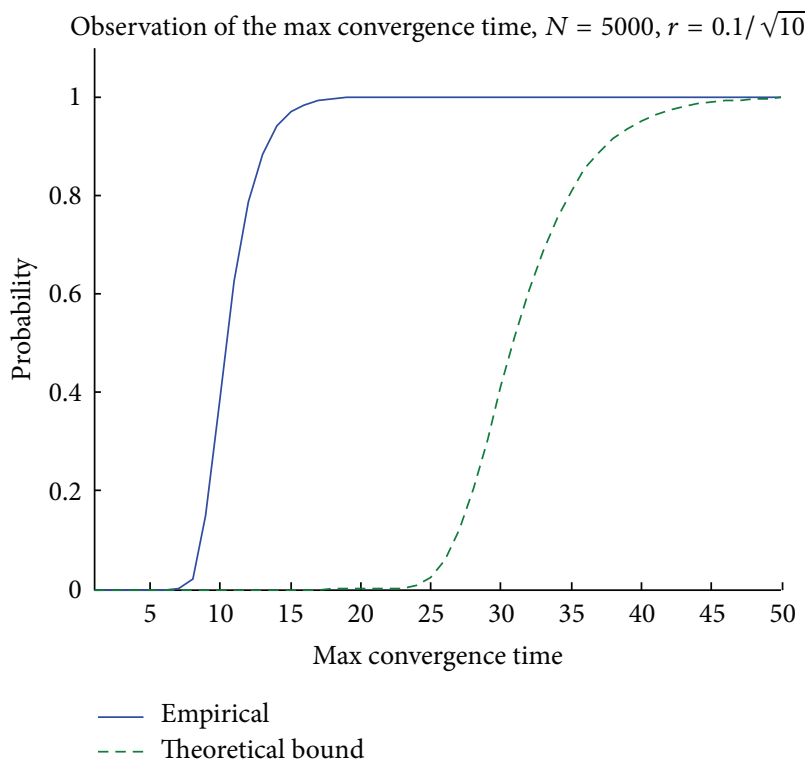

(c)

FIGURE 2: Numerical validation of Theorem 7's bound on the network-wise convergence time. We compare the bound, $P\left(t_{\max }<k\right)=(1-$ $\left.(1-q)^{k}\right)^{N}$, with the numerical results, which consider random geometric graphs in which the nodes are randomly placed on the unit square. The charts considers $N \in\{500,2500,5000\}$ nodes (from left to right). All experiments considered 2 listening/signaling periods, interference range of $0.1 / \sqrt{(N / 500)}$, which result in an average extended degree of $15, d_{i} / T=1$ on average, and $q_{i}=1 / 4$. 


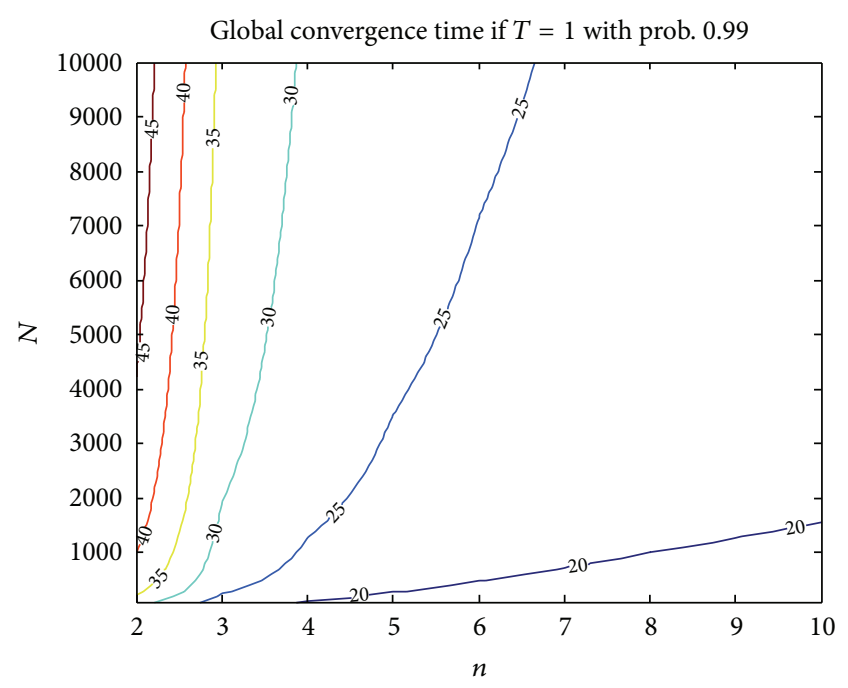

Figure 3: Contour plot of (22) for $s=d / T=1$. Contour charts [50] present two parameter functions, for example, the convergence time function, $k(n, N)$ presented in (22). Contour lines in Figure 3 connect values of $k(n, N)$ that are the same (see the text tags along the line). When $N$ nodes attempt to access the medium, the convergence time, $\mathcal{S}$ (cf. the contour lines), is stable in the presence of a growing number, $n$, of listening/signaling periods.

uses beacons for signaling the node's intention to transmit a DATA packet within $t$; see Figure 1.

2.4. System Settings. We consider the interleaving model [4]. Every node, $p_{i} \in P$, executes a program that is a sequence of atomic steps. The state $s t_{i}$ of a node $p_{i}$ consists of the value of all the variables of the node (including messages in transit for $p_{i}$ ). Variables are associated with individual node states by using the subscript notation, that is, $v a r_{i}$ is the value of variable var in $p_{i}$ 's state. The term configuration is used for a tuple of the form $\left(G,\left\{s t_{i}\right\}_{i=1}^{N}\right)$, where $G$ is the (interference) graph, and $\left\{s t_{i}\right\}_{i=1}^{N}$ are the nodes' states (including the set of all incoming communications). An execution (run) $R:=$ $(c(0), c(1), \ldots)$ is an unbounded sequence of system configurations $c(x)$, such that each configuration $c(x+1)$ (except the initial configuration $c(0))$ is obtained from the preceding configuration $c(x)$ by the execution of steps, $\left\{a_{i}(x)\right\}_{p_{i} \in P}$, taken by all nodes.

Let $\tau$ (task) be a specification (predicate) set and $L E$ a set of all executions that satisfy task $\tau$. Let us consider TDMAbased MAC protocols for which the task $\tau_{\text {TDMA }}$ requires that every node has its own broadcasting timeslot that is unique within its neighborhood. We note that $\tau_{\text {TDMA }}$ 's requirements are obviously satisfiable when the ratio between the extended degree and the frame size is less than one; that is, there is no timeslot exhaustion when for all $p_{i} \in P: 1 \lessgtr T / d_{i}$. Therefore, the studied task also deals with timeslot exhaustion by delivering busy channel indications, $\perp$, to the nodes for which there were no timeslot left. We define $L E_{\mathrm{TDMA}}$ to be the set of legal executions, $R$, for which for all $p_{i} \in P:\left(\left(s_{i} \in\right.\right.$ $\left.\left.[0, T-1]) \wedge\left(p_{j} \in \mathcal{N}_{i}\right)\right) \Rightarrow s_{i} \neq s_{j}\right) \vee\left(s_{i}=\perp \Rightarrow\right.$ for all $t \in$ $\left.[0, T-1] \exists p_{j} \in \mathcal{N}_{i}: s_{j}=t\right)$ holds in all of R's configurations.
We say that configuration $c_{\text {safe }}$ is safe if there is an execution $R \in L E$, such that $c_{\text {safe }}$ is $R$ 's starting configuration. Let $R$ be an execution and $c \in R$ its arbitrary starting configuration. We say that $R$ converges with respect to $\tau$ if within a bounded number of steps from $c$, the system reaches a safe configuration $c_{\text {safe }}$. The closure property requires that $R \in L E$, for any execution, $R$, that starts form $c_{\text {safe }}$. An algorithm is said to be self-stabilizing if it satisfies both the convergence and the closure properties.

We describe execution $R$ as an unbounded number of concatenated finite sequences of configurations. The finite sequence, $R(x)=\left(c_{0}(x), \ldots c_{T-1}(x)\right), x>0$, is a broadcasting round if (1) configuration $c_{0}(x)$ has a clock value, $t$, of 0 and immediately follows a configuration in which the clock value is $T-1$, and (2) configuration $c_{T-1}(x)$ has a clock value of $T-1$ and immediately precedes a configuration in which the clock value is 0 .

\section{Algorithm Description}

The proposed MAC algorithm periodically performs clear channel assessments. It uses these assessments when informing each node about the nearby unused timeslots. The nodes use this information for selecting their broadcasting timeslots, assessing the success of their broadcasts and reselecting timeslots when needed.

The MAC algorithm in Algorithm 1 satisfies the $\tau_{\text {TDMA }}$ task. During the convergence period, several nodes can be assigned to the same timeslot. Namely, we may have $p_{i} \in P: p_{j} \in \mathcal{N}_{i} \wedge s_{i}=s_{j}$. The algorithm solves such timeslot allocation conflicts by letting the nodes $p_{i}$ and $p_{j}$ go through a (listening/signaling) competition before transmitting in its broadcasting timeslot. The competition rules require each node to choose one out of MaxRnd listening/signaling periods for its broadcasting timeslot; see Figure 1. This implies that among all the nodes that attempt to broadcast in the same timeslot, the ones that select the earliest listening/signaling period win this broadcasting timeslot and access the communication media. Before the winners access their timeslots, they signal to their neighbors that they won via beacon transmission. The signal is sent during their choice of listening/signaling periods; see Figure 1. When a node receives a beacon, it does not transmit during that timeslot, because it lost this (listening/signaling) competition. Instead, it randomly selects another broadcasting timeslot and competes for it on the next broadcasting round.

In detail, the MAC algorithm in Algorithm 1 is invoked at the start of every timeslot, $t$. When $t$ is the first timeslot, the algorithm tries to allocate the broadcasting timeslot, $s_{i}$, to $p_{i}$ (line 11) by randomly selecting a timeslot for which there is no indication to be used by its neighbors. Later, when the timeslot $t$ becomes $p_{i}$ 's broadcasting timeslot, $s_{i}$, the node attempts to broadcast (by calling the function send() in line 13). We note that the start of timeslot $t$ also requires the marking of $t$ as an unused timeslot and the removal of stale information (line 12). This indication is changed when the carrier_sense $(t)$ event is raised (line 27) due to a neighbor transmission; namely, when the detected energy levels reach 


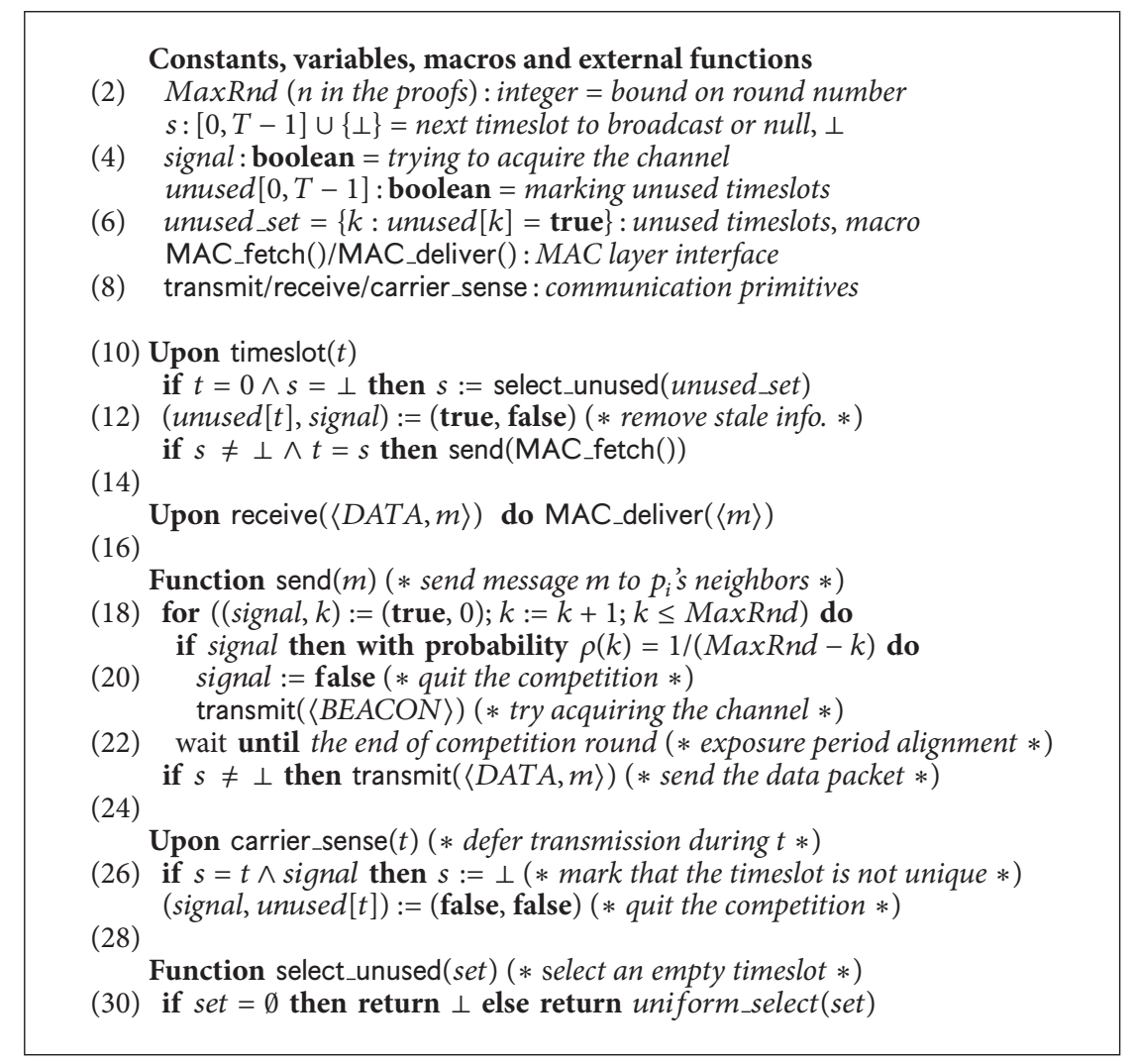

Algorithm 1: Self-stabilizing TDMA-based MAC algorithm, code of node $p_{i}$.

a threshold in which the radio unit is expected to succeed in carrier locking; see [45].

When a node attempts to broadcast, it uses the (listening/signaling) competition mechanism for deciding when to signal to its neighbors that it is about to transmit a DATA packet. The competition has MaxRnd rounds, and it stops as soon as the node transmits a beacon or a neighbor succeeds in signaling earlier (lines 18 to 23). We note that this signaling is handled by the carrier_sense $(t)$ event (line 27). Moreover, beacons are not required to carry payloads or any other information that is normally stored in packet headers. They are rather used to invoke the carrier sense event in $\mathcal{N}_{i}$.

The carrier sense in timeslot $t$ indicates to each node that it needs to defer from transmission during $t$ (line 25). In particular, it should stop using timeslot $t$ for broadcasting, stop competing, and mark $t$ as a used timeslot. Lastly, arriving DATA packets are delivered to the upper layer (line 15).

\section{Correctness Proof: Outline and Notation}

The proof starts by considering networks that do not change their topology and for which the ratio between the extended node degree and the frame size is less than one, that is, for all $p_{i} \in P: 1 \lessgtr T / d_{i}$. (We deal with cases in which for all $p_{i} \in P: 1 \subsetneq T / d_{i}$ does not hold in Section 8). For these settings, we show that the MAC algorithm in Algorithm 1 is self-stabilizing with respect to task $\tau_{\text {TDMA }}$ (Appendices $\mathrm{A}$ to $\mathrm{B}$ ), before considering the convergence time within a single neighborhood (Section 5) and the entire neighborhood (Section 6). These convergence estimations facilitate the exploration of important properties, such as predictability, and dealing with changes in the network topology of DynWANs (Section 8).

4.1. Proof Outline. The exposition of the proof outline refers to Definition 1, which delineates the different states at which a node can be in relation to its neighbors. Definition 1 groups these states into three categories of relative states: (1) Ready to be allocated, when the node state depicts correctly its neighbor states, (2) Obtaining a timeslot, when the node is competing for one, but there is no agreement with its neighbor states, and (3) Allocated to a timeslot, when the node is the only one to be allocated to a particular timeslot in its neighborhood. The correctness proof shows that the MAC algorithm in Algorithm 1 implements $\tau_{\mathrm{TDMA}}$ in a selfstabilizing manner by showing that eventually all nodes are allocated with timeslots; that is, all nodes are in the relative state Allocated; see Definition 1.

Let $R$ be an execution of the MAC algorithm in Algorithm 1 and $R(x)$ the $x$ th complete broadcasting round of $R$, where $x>0$ is an integer. We simplify the presentation by using uppercase notation for the configurations, $c_{t}^{\text {name }}(x)$, where $t \in[0, T-1]$ is a timeslot. This notation includes the name of the first event to be triggered immediately after configuration $c$, that is, $R(x)=\left(c_{0}^{\text {timeslot }}(x), \ldots c_{T-1}^{\text {carrier_sense/receive }}(x)\right)$. 
Definition 1. We say that node $p_{i} \in P$ is Ready (to be allocated) to a timeslot in configuration $c_{0}^{\text {timeslot }}(x)$, if properties $(1),(2)$, and (3) hold for node $p_{i}$, but Property (4) does not. We say that $p_{i}$ is Obtaining timeslot $s_{i}$ in configuration $c_{0}^{\text {timeslot }}(x)$, if properties (1) to (4) hold for node $p_{i}$, but Property (5) does not. We say that node $p_{i} \in P$ is in Allocated state, with respect to timeslot $s_{i}$ in configuration $c_{0}^{\text {timeslot }}(x)$, if properties (1) to (5) hold for node $p_{i}$ as follows:

$$
\begin{gathered}
\text { signal }_{i}=\text { false } \\
\left(t \in \text { unused }_{i} \wedge t \neq s_{i}\right) \longleftrightarrow\left(\forall p_{k} \in \mathcal{N}_{i}: s_{k} \neq t\right) \\
s_{i} \neq \perp \vee \text { unused_set } \backslash\left\{s_{i}\right\} \neq \emptyset \\
s_{i} \neq \perp \\
\forall p_{j} \in \mathcal{N}_{i}:\left(\left(s_{i} \neq s_{j}\right) \wedge\left(\text { unused }_{j}\left[s_{i}\right]=\text { false }\right)\right) .
\end{gathered}
$$

Property (1) implies that node $p_{i}$ finishes any broadcast attempts within a timeslot. Properties (2) to (3) consider the case in which $p_{i}$ 's internal state represents correctly the timeslot allocation in its neighborhood. In particular, property (2) means that processor $p_{i}$ views timeslot $t$ as an unused one if, and only if, it is indeed unused. Property (3) implies that when node $p_{i}$ is not using any timeslot, there is an unused timeslot at its disposal. Property (4) says that node $p_{i}$ is using timeslot $s_{i}$. Property (5) refers to situations in which $p_{i}$ 's neighbors are not using $p_{i}$ 's timeslot during the next broadcasting round.

Starting from an arbitrary configuration, we show that node $p_{i}$ becomes Ready within two broadcasting rounds (or one complete broadcasting round); see Appendix A. Then, we consider the probability, OnlyOne ${ }_{i}(x)$, that a node enters the relative state Allocated from either Ready or Obtaining; see (6) (and Appendices B and D). Namely, (6) considers the probability that node $p_{i}$ is the only one to use its broadcasting timeslot in its neighborhood, where $\rho_{k}=1 / \operatorname{MaxR} n d=1 / n$ is $p_{i}$ 's probability to select the $k$ th listening/signaling period for transmitting its beacon.

Consider

$$
\operatorname{OnlyOne}_{i}(x) \geq \sum_{k=1}^{n} \rho_{k}\left(1-\sum_{\ell=1}^{k} \rho_{\ell}\right)^{d_{i} / T} .
$$

Theorem 2 demonstrates self-stabilization.

Theorem 2 (self-stabilization, the proof appears in Appendix C). The MAC algorithm in Algorithm 1 is selfstabilizing with respect to the task $\tau_{\text {TDMA }}$.

Bounding the convergence time. We bound the time it takes the MAC algorithm in Algorithm 1 to converge by considering the relative states, Ready, Obtaining, and Allocated and describe a state machine of a Markovian process. This process is used for bounding the convergence time of a single node (Section 5) and the entire network (Section 6).

In detail, given node $p_{i} \in P$, its neighborhood $\mathcal{N}_{i}$, we define a random environment of a Markov chain; see Box 1 .
By looking at this random environment, we can focus our analysis on $p_{i}$ 's relative states while avoiding probability dependencies and considering average probabilities [46]. Suppose that $p_{i}$ 's environment, $e$, is known. Theorem 3 estimates two bounds on the expectation of probability $\left.q_{i}\right|_{e}$, which is literally the probability $q_{i}$, given that the environment is $e$.

In order to do that, we consider a set, $\mathscr{R}$, of executions of the MAC algorithm, such that each execution $R \in \mathscr{R}$ starts in a configuration, $c \in R$, in which (I) for any node $p_{j} \in P$, properties (1), (2), and (3) hold, and (II) node $p_{i}$ is in the relative state Ready, which implies that (III) eventually, node $p_{i}$ arrives to the relative state Allocated.

With this convention, we can add a probability 1 to transit from the relative state Allocated to Ready; see the dashed line in the state machine diagram of Box 1 . This allows us to estimate the expected time to reach the final relative state Allocated from relative state Ready by the expectation of the first hitting time of the irreducible Markov chain [43].

When computing the expected time for node $p_{i}$ to reach state Allocated within its neighborhood, we see that it is sufficient to consider the lower bound of the probability $\mathrm{OnlyOne}_{i}(x)$ to obtain an upper bound on the expected time to convergence; see Section 5. Moreover, when considering the network convergence time, that is, the expected convergence time of all nodes in the network, we see that the most dominant parameter is the mean neighborhood size. We do that by applying the arithmetic mean versus geometric mean (AM-GM) inequality and bounding the expected network convergence time; see Section 6.

4.2. Notation. Throughout the paper, we denote the states of the Markov chain by $\left\{X_{t}\right\}_{t \geq 0}, T_{i}^{+}=\min \left\{t>0\right.$ such that $X_{t}=$ $i\}$ and $E_{i}(\cdot)$ is the expectation, given that we start in relative state $i, E_{i}\left(T_{i}^{+}\right)=E\left(T_{i}^{+} \mid X_{0}=i\right)$. In this paper, the states 1 , 2 , and 3 of the Markovian process correspond, respectively, to states Ready, Obtaining and Allocated and the time $t=0,1, \ldots$ corresponds to configuration $c_{0}^{\text {timeslot }}(x+t) \in R(x+t)$, where $R(x)$ is the first complete broadcasting round in $R$ that starts in a configuration, $c_{0}^{\text {timeslot }}(x)$, in which all nodes are in the relative state Ready. For example, $E_{3}\left(T_{3}^{+}\right)$is the expected time to reach the Allocated state.

Let $p_{i} \in P$ be a node for which $s_{i} \neq \perp \wedge \exists p_{j} \in \mathcal{N}_{i}$ : $s_{j}=s_{i}$ in configuration $c_{0}^{\text {timeslot }}(x)$. We define $M_{i}(x)=\left\{p_{j} \in\right.$ $\left.\mathcal{N}_{i}: s_{i}=s_{j}\right\}$ to be the set of $p_{i}$ 's (broadcasting timeslot) matching neighbors, which includes all of $p_{i}$ 's neighbors that, during broadcasting round $R(x)$, are attempting to broadcast in $p_{i}$ 's timeslot. In our proofs, we use $n$ as the number of listening/signaling periods, MaxRnd.

\section{Convergence within a Neighborhood}

Theorem 3 bounds the expected time, $\mathcal{S}_{i}$, for a node to reach the relative state Allocated, and follows from Proposition 5 and (12). Note that $\mathcal{S}_{i} \leq 4$ when the number of listening/signaling periods is $n \geq 2$ and considering saturated situations in which the extended node degree $d_{i}<T$ is 


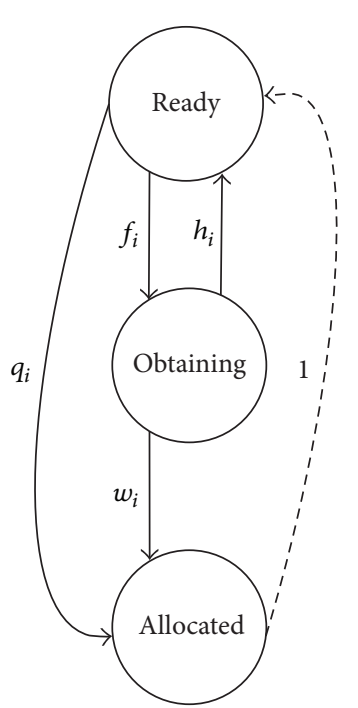

We look at $p_{i}$ 's state transition with relation to its neighbors; see Definition 1 . The figure on the top defines $p_{i}$ 's relative states as a 3 -state Markov chain. The probabilities, $q_{i}, w_{i}, f_{i}$, and $h_{i}$ (solid lines arrows), that node $p_{i}$ change its relative state depend on its neighbor's state. For instance, $q_{i}$ is the probability that $p_{i}$ goes from the relative state Ready to Allocated. It is environmentdependent; that is, the states of $p_{i}$ 's neighbors are random as well. We added the dotted edge between the state Allocated and the state Ready in order to make the Markov chain irreducible and to allow working with the invariant probability. Namely, once node $p_{i}$ arrives to Allocated, it returns to Ready with probability 1 . With this convention, we can estimate the expected time to reach the final relative state Allocated from relative state Ready by the expectation of the first hitting time of the irreducible chain [43]

Box 1: Markov chain describing $p_{i}$ 's relative state transitions.

smaller than the TDMA frame size. Namely, the proposed algorithm convergence with a neighborhood is brief.

Theorem 3 (local convergence). The expected time, $\mathcal{S}_{i}$, for node $p_{i} \in P$ to reach the relative state Allocated satisfies (7), where $n$ is the number of listening/signaling periods, $T$ is the TDMA frame size, and $d_{i}$ is $p_{i}$ 's extended degree.

Consider

$$
\mathcal{S}_{i} \leq \min \left\{\left(\frac{2 n}{n-1}\right)^{d_{i} / T}, \frac{d_{i} / T+1}{n}\left(\frac{n}{n-1}\right)^{d_{i} / T+1}\right\} .
$$

We look into the transition probability among relative states by depicting the diagram of Box 1 as a homogeneous Markov chain. We estimate the diagram transition probabilities in a way that maximizes the expected time for reaching the diagram's final state, Allocated. It is known that the first hitting time is given by $E_{i}\left(T_{i}^{+}\right)=1 / \pi_{i}$, where $\pi=\left(\pi_{1}, \pi_{2}, \pi_{3}\right)$ is the invariant probability vector [43]. Let $\mathcal{S}_{i}$ be the expected time it takes node $p_{i}$ that starts at the relative state Ready to reach Allocated. It is clear that $\mathcal{S}_{i}=T_{3}^{+}-1$, because $T_{3}^{+}-1$ is the return time of the relative state Allocated. In our case, the transition matrix $P$ is given by the following:

$$
P=\left(\begin{array}{ccc}
1-f_{i}-q_{i} & f_{i} & q_{i} \\
h_{i} & 1-h_{i}-w_{i} & w_{i} \\
1 & 0 & 0
\end{array}\right) .
$$

The invariant probability vector $\pi$ satisfying $\pi P=\pi$ is given by

$$
\pi=\frac{\left(h_{i}+w_{i}, f_{i}, q_{i} h_{i}+q_{i} w_{i}+f_{i} w_{i}\right)}{h_{i}+w_{i}+f_{i}+h_{i} q_{i}+q_{i} w_{i}+f_{i} w_{i}} .
$$

The estimation of the maximal expected time necessary to assign the node $p_{i}$ to a timeslot requires us to compute bounds on the probabilities $f_{i}, h_{i}, q_{i}$ and $w_{i}$ that maximize as follows

$$
E_{3}\left(T_{3}^{+}\right)=\frac{1}{\pi_{3}}=\frac{h_{i}+w_{i}+f_{i}+h_{i} q_{i}+q_{i} w_{i}+f_{i} w_{i}}{q_{i} h_{i}+q_{i} w_{i}+f_{i} w_{i}} .
$$

The expected time for $p_{i}$ to reach the relative state Allocated is bounded in

$$
\mathcal{S}_{i}=E_{3}\left(T_{3}^{+}\right)-1=\frac{h_{i}+w_{i}+f_{i}}{q_{i} h_{i}+q_{i} w_{i}+f_{i} w_{i}} .
$$


Equation (7) has a compact and meaningful bound for (11). We achieve that by studying the impact of the parameters $T$ and $n$ on the MAC algorithm in Algorithm 1. Lemma 4 and (11) imply

$$
\mathcal{S}_{i} \leq \frac{h_{i}+w_{i}+f_{i}}{q_{i} h_{i}+q_{i} w_{i}+f_{i} q_{i}}=\frac{1}{q_{i}} .
$$

Lemma 4. Suppose that $n \geq 2$ is the number of listening/signaling periods; see line 2 of the code in Algorithm 1. Then $w_{i} \geq q_{i}$.

Proof. Let us consider node $p_{i} \in P$ that is in relative state Ready. Given that $p_{i}$ has $v_{i}$ neighbors that compete for the same timeslot, the probability that $p_{i}$ gets allocated, $\left.q_{i}\right|_{v_{i}}$, is given by (13).

$$
\left.q_{i}\right|_{v_{i}}=\sum_{k=1}^{n-1} \rho_{k}\left(1-\rho_{1}-\cdots-\rho_{k}\right)^{v_{i}} .
$$

Consider next that $p_{i}$ is in relative state Obtaining, and thus, we know that $p_{i}$ transmitted during the preceding broadcasting round and transited from relative state Ready to Obtaining. Moreover, $p_{i}$ is using the same timeslot for the current broadcasting round. The only neighbors of $p_{i}$ that are using the same timeslot are the neighbors that are also in relative state Obtaining and have chosen the same listening/signaling period as $p_{i}$ during the preceding broadcasting round. Let us denote by $\ell_{i}$, the number of such neighbors. Given $\ell_{i}$ the probability $\left.w_{i}\right|_{\ell_{i}}$ that $p_{i}$ is allocated to the timeslot is given by

$$
\left.w_{i}\right|_{\ell_{i}}=\sum_{k=1}^{n-1} \rho_{k}\left(1-\rho_{1}-\cdots-\rho_{k}\right)^{\ell_{i}} .
$$

We have that $\ell_{i}$ is stochastically dominated by $v_{i}$ [47], that is, $E\left(\ell_{i}\right) \leq E\left(v_{i}\right)$. Indeed, $v_{i}$ is a random variable that counts the number of neighbors that choose the same timeslot as $p_{i}$, while $\ell_{i}$ counts the number of neighbors that choose the same timeslot and listening/signaling period as $p_{i}$. For $n \geq$ $2, \ell_{i}$ 's expected value is smaller than $v_{i}$ 's expected value. To conclude, we remark that expressions (13) and (14) are the same decreasing function, $f_{i} \rightarrow \sum_{k=1}^{n-1} \rho_{k}\left(1-\rho_{1}-\cdots-\rho_{k}\right)^{f_{i}}$, that is evaluated at two different points, $v_{i}$ and $\ell_{i}$, respectively. Moreover, since $\ell_{i}$ is stochastically dominated by $v_{i},(15)$ holds as follows:

$$
w_{i}=E\left(\left.w_{i}\right|_{\ell_{i}}\right) \geq E\left(\left.q_{i}\right|_{v_{i}}\right)=q_{i} .
$$

Proposition 5 demonstrates (16) and leads us toward the proof of Theorem 3 .

Proposition 5. Let $\rho_{i}=1 /$ MaxRnd. Equation (16) bounds from below the probability $q_{i}$; see Appendix $D$.

Consider

$$
q_{i} \geq \max \left\{\left(\frac{n-1}{2 n}\right)^{d_{i} / T}, \frac{1}{d_{i} / T+1}\left(1-\frac{1}{n}\right)^{d_{i} / T+1}\right\} .
$$

The first bound, $1 / q_{i} \leq(2 n /(n-1))^{d_{i} / T}((7))$, has a simple intuitive interpretation. Let us consider first that two nodes compete for a same timeslot. The two nodes choose independently any of the $n$ listening/signaling periods and there are $n^{2}$ different possible outcomes. Among these outcomes $n$ correspond to the situation where the two nodes choose the same listening/signaling period and there is no winner. We then have $n^{2}-n=n(n-1)$ outcomes that lead to a winner. There is then a probability of $n(n-1) / n^{2}=$ $(n-1) / n$ that one of the nodes wins the (listening/signaling) competition. Since the game is symmetric, the probability that $p_{i}$ wins is $(n-1) /(2 n)$. The fact that we have $T$ timeslots divides the number of competing nodes, $d_{i}$, and implies that there are $d_{i} / T$ competing nodes for the same timeslot. If we interpret the game as a collection of $d_{i} / T$ independent games, where for each game $p_{i}$ wins with probability $(n-1) /(2 n)$, thus, the probability $q_{i}$ that $p_{i}$ wins is $((n-1) / 2 n)^{d_{i} / T}$. The inverse of this expression gives the average time for the event to occur and is the bound by (7).

\section{Network Convergence}

We estimate the expected time for the entire network to reach a safe configuration in which all nodes are allocated with timeslots. The estimation is based on the number of nodes that are the earliest to signal in their broadcasting timeslot. These nodes are winners of the (listening/signaling) competition and are allocated to their chosen timeslots. However, counting only these nodes leads to underestimating the number of allocated nodes, which then results in an overestimation of the convergence time. Indeed, node $p_{i} \in$ $P$ might have a neighbor $p_{j} \in \mathcal{N}_{i}$ that selects the earliest listening/signaling period in $\mathcal{N}_{i}$, but $p_{j}$ does not transmit because one of its neighbors, $p_{k} \in \mathcal{N}_{j} \backslash \mathcal{N}_{i}$, had transmitted in an earlier listening/signaling period. Our bound considers only $p_{k}$ while both $p_{i}$ and $p_{k}$ transmit, became $p_{j}$ is inhibited by $p_{k}$ 's beacon.

Lemma 6 shows that the assumption that the nodes are allocated independently of each other is suitable for bounding the network convergence time, $\mathcal{S}$. Theorem 7 uses Lemma 6 for bounding the network convergence time, $\delta$.

In Section 5, we prove a bound on the expected time, $\mathcal{S}_{i}$, for a single node to be allocated to a timeslot. We observe that the bound depends uniquely on the number of listening/signaling periods, $n$, as well as the ratio between the extended degree and the frame size, $d_{i} / T$. In order to obtain a bound valid for all nodes, we bound this ratio with $x / T$ where $x$ is as defined in Lemma 6 . We note that the time needed for the allocation of timeslots to all the nodes depends on $N$, the total number of nodes.

In detail, the convergence time estimation considers the (fixed and independent) bound, $q_{i}$, for the probability that a node reaches the relative state Allocated within a broadcasting round. Then, the convergence time, $t$, is a random variable with geometric probability, that is, $P(t=k)=(1-$ $q)^{k-1} q$. Let us denotes $t_{1}, \ldots, t_{N}$ the time it takes for the nodes $p_{1}, \ldots, p_{N}$ to respectively reach the relative state Allocated. 
The convergence time, $\mathcal{S}$, for all the nodes is given with $\max \left(\left\{t_{1}, \ldots, t_{N}\right\}\right)$, which depends on $N$.

Lemma 6. The expected number of nodes, $E(W)$, that win the (listening/signaling) competition after one broadcasting round satisfies (17), where $x=2 A / N, T$ is the number of timeslots, $A$ the number of edges in the interference graph, $G$, and $N=|P|$ the number of nodes that attempt to access the communication media.

Consider

$$
E(W) \geq N \sum_{j=1}^{n} \rho_{j}\left(1-\left(\rho_{1}+\cdots+\rho_{j}\right)\right)^{x / T}
$$

Proof. The nodes that are allocated to a timeslot can previously be on relative state Ready or Obtaining. The probability of a transition from relative state Obtaining to Allocated is $w_{i}$, and a transition from relative state Ready to Allocated is $q_{i}$. As proved in Lemma 4 , we always have $w_{i} \geq q_{i}$. To bound the number of nodes that get allocated during a broadcasting round, we use the lower bound on the probability $q_{i}$ that a node gets allocated to a timeslot. Moreover, in the computations, we use the AM-GM bound [48], which says that if $\sum b_{k}=1$ then $\prod a_{k}^{b_{k}} \leq \sum b_{k} a_{k}$ and denote by $d_{i}$ the number of neighbors of node $p_{i}$. As proved in Proposition D.1, since there are $T$ timeslots the number of neighbors of $i$ that choose the same timeslot as $i$ and compete for it is bounded by $d_{i} / T$. This lemma is proved by (18), where the last line of the expression holds because $\sum_{i} d_{i}=2 A$.

One has

$$
\begin{aligned}
E(W) & \geq E\left(\sum_{i=1}^{N} 1_{\left.\mid p_{i} \text { selects the earliest signaling period }\right)}\right. \\
& =\sum_{i=1}^{N}\left(\rho_{1}\left(1-\rho_{1}\right)^{d_{i} / T}+\cdots \rho_{n-1}\left(1-\sum_{k=1}^{n-1} \rho_{k}\right)^{d_{i} / T}\right) \\
& =\sum_{j=1}^{n} N \sum_{i=1}^{N} \frac{1}{N} \rho_{j}\left(1-\sum_{k=1}^{j} \rho_{k}\right)^{d_{i} / T} \\
& \geq N \sum_{j=1}^{n} \prod_{i=1}^{N} \rho_{j}^{1 / N}\left(1-\sum_{k=1}^{j} \rho_{k}\right)^{d_{i} / N T} \\
& =N \sum_{j=1}^{n} \rho_{j}\left(1-\sum_{k=1}^{j} \rho_{k}\right)^{(1 / T N) \sum d_{i}} \\
& =N \sum_{j=1}^{n} \rho_{j}\left(1-\sum_{k=1}^{j} \rho_{k}\right)^{x / T} .
\end{aligned}
$$

We note that we use the AM-GM bound to reach the 4th row of (18).

By arguments similar to the ones used in the proof of Proposition 5, we deduce that if $N$ nodes compete, the expected number $E(W)$ of nodes that get allocated to a timeslot is lower bounded as follows:

$$
E(W) \geq N \max \left\{\left(\frac{n-1}{2 n}\right)^{x / T}, \frac{((n-1) / n)^{x / T+1}}{x / T+1}\right\} .
$$

Theorem 7 bounds the system convergence time. We numerically validate Theorem 7; see Figure 2. Moreover, our experiments showed that the average convergence time of the network is below the upper bound of (21).

Theorem 7 (global convergence). The expected number of retransmissions is smaller than $(2 n /(n-1))^{d / T}-1$, where $d=$ $\max \left(\left\{d_{i}: p_{i} \in P\right\}\right)$. Hence, one has that the expected number of broadcasting rounds, $\mathcal{S}$, that guarantee that all nodes reach the relative state Allocated satisfies

$$
\mathcal{S} \leq\left(\frac{2 n}{n-1}\right)^{d / T} .
$$

Moreover, given that there are $N$ nodes in the network and $\alpha \in(0,1)$, the network convergence time is bounded by (22) with probability $1-\alpha$.

Consider

$$
k=1+\frac{\log (1-\sqrt[N]{1-\alpha})}{\log \left(1-((n-1) / 2 n)^{d / T}\right)}
$$

This means that with probability $\alpha$, all nodes are allocated with timeslots in maximum $k$ broadcasting rounds; see Figure 3.

Proof. Theorem 3 bounds the convergence time of a particular processor; see (7). Lemma 6; see (20) $E(W) \geq N((n-$ $1) / 2 n)^{x / T}$, proves that this bound is still valid if we replace the term $d_{i} / T$ with $x / T$; that is, we consider the average degree instead of the particular degree of a node. If we replace $x / T$ by $\max \left\{d_{i}\right\} / T$ in expression (20) we obtain a larger bound because $x / T \leq \max \left\{d_{i}\right\} / T$; that is, $E(W) \geq N((n-1) / 2 n)^{x / T} \geq$ $N((n-1) / 2 n)^{\max \left\{d_{i}\right\} / T}$.

The bound $E(W) \geq N((n-1) / 2 n)^{\max \left\{d_{i}\right\} / T}$ and the dis- cussion in the 1st paragraph of Section 6 show that the number of processors that are allocated during a broadcasting round is bounded by the random variable $\sum_{i=1}^{N} z_{i}$, where $z_{i}$ are identically and independently distributed random variables that are 1 with probability $((n-1) / 2 n)^{\max \left\{d_{i}\right\} / T}$ and 0 with probability $1-((n-1) / 2 n)^{\max \left\{d_{i}\right\} / T}$ (the second random variable dominates the first one; see [49]). This means that we lower bound the number of processors that are allocated if we consider that they are allocated independently with probability $((n-1) / 2 n)^{\max \left\{d_{i}\right\} / T}$.

While the processors get allocated to a timeslot, the parameters $d_{i}$ and $T$ change because some timeslots are no longer available ( $T$ decreases, some nodes are allocated, $d_{i}$ decreases). Actually the ratio becomes $\left(\max \left\{d_{i}\right\}-h_{i}\right) /\left(T-f_{i}\right)$, where $h_{i} \geq f_{i}$ because if a timeslot is allocated or sensed used by processor $p_{i}$, then $T$, the number of available timeslots decreases by 1 and $d_{i}$, the number of competing nodes, must decrease at least by one since there must be at least one 
processor that uses the busy timeslot (there may be multiple that are in state Obtaining). Under these circumstances, we always have $\max \left\{d_{i}\right\} / T \geq\left(\max \left\{d_{i}\right\}-h_{i}\right) /\left(T-f_{i}\right)$. Thus, we can obtain a lower bound for the expected time to reach the relative state Allocated by assuming that all nodes are allocated independently with probability $x=((n-$ $1) / 2 n)^{\max \left\{d_{i}\right\} / T}$. We simplify the following arguments by using this definition of $x$.

To bound the number of broadcasting rounds, we consider the following game. The bank pays 1 unit to the nodes that get in state Allocated (get allocated to a timeslot) and receives $x /(1-x)$ units per nodes that fail to get in state Allocated. The game is fair because in each round the expected gain is $1 \times x-x /(1-x) \times(1-x)=0$. If we denote by $W_{i}$ the number of processors that get in state Allocated during the $i$ th broadcasting round and by $L_{i}$ the number of processors that fail, we have that the gain is given by (23), where $t$ denotes the total number of rounds.

One has

$$
\text { gain }=\sum_{i=1}^{t}\left(\frac{x}{1-x} L_{i}-W_{i}\right)
$$

The expected gain is 0 because the game is fair $(E$ (gain) $=0)$ and $\sum_{i=1}^{t} W_{i}=N$ because eventually all the nodes get in state Allocated and the bank pays 1 unit for each such processors. If we compute the expectation on both sides of (23), we then obtain

$$
N=\frac{x}{1-x} E\left(\sum_{i=1}^{t} L_{i}\right) .
$$

We observe that $E\left(\sum_{i=1}^{t} L_{i}\right)$ is the expected total number of retransmissions and $E\left(\sum_{i=1}^{t} L_{i}\right) / N$ is the average expected number of retransmissions whose value is $(1-x) / x$. Replacing $x$ with its expression, we obtain that the average number of retransmission is bounded by $(2 n /(n-1))^{\max \left\{d_{i}\right\} / T}-1$, and, this leads to the bound (21).

To prove the second assertion, let $t_{1}, \ldots, t_{N}$ be the convergence time of nodes $1, \ldots, N$, respectively. The random variables, $t_{i}$, are bound by random variables with geometric random distribution with expectation of $(2 n /(n-1))^{d / T}$, with $d=\max \left\{d_{i}: d_{i} \in P\right\}$. We require that $t_{\max }=\max \left\{t_{1}, \ldots, t_{N}\right\}$ in order to ensure that all nodes are allocated with timeslots. The fact that the random variables, $t_{i}$, are independent and identically distributed, implies (25), where $t$ is a random geometrical random variable, that is, $\operatorname{Pr}\left(t=k^{\prime}\right)=(1-q)^{k^{\prime}-1} q$ and $\operatorname{Pr}\left(t \geq k^{\prime}\right)=(1-q)^{k^{\prime}-1}$.

Consider

$$
\begin{aligned}
\operatorname{Pr}\left(t_{\max } \leq k^{\prime}\right) & =P\left(t_{1} \leq k^{\prime}, \ldots, t_{N} \leq k^{\prime}\right) \\
& =\operatorname{Pr}\left(t_{1} \leq k^{\prime}\right) \cdots \cdot P\left(t_{N} \leq k^{\prime}\right) \\
& =P\left(t \leq k^{\prime}\right)^{N} .
\end{aligned}
$$

Which $t_{\max } \leq k^{\prime}$ satisfies (26) with probability $\alpha$ ?
One has

$$
\begin{aligned}
\operatorname{Pr}\left(t_{\max }<k^{\prime}\right) & =\operatorname{Pr}\left(t<k^{\prime}\right)^{N} \\
& =\left(1-(1-q)^{k^{\prime}-1}\right)^{N} \geq 1-\alpha
\end{aligned}
$$

By solving (26), we observe that (26) is satisfied for any $k^{\prime} \geq k$, where $k$ satisfies (22). This proves that, with probability $1-\alpha$, the network convergence time is bounded by (22).

\section{Implementation}

Existing MAC protocols offer mechanisms for dealing with contention (timeslot exhaustion) via policies for administering message priority, such as [13]. In particular, the IEEE $802.11 \mathrm{p}$ standard considers four priorities and techniques for facilitating their policy implementation. We explain similar techniques that can facilitate the needed mechanisms.

7.1. Prioritized Listening/Signaling Periods. We partition the sequence of listening periods, [0, MaxRnd), into MaxPrt subsequences, [0, MaxRnd $\left.d_{0}\right)_{, \ldots}\left[\right.$ MaxRnd $_{\text {MaxPrt-2 }}$, $\left.\operatorname{MaxRnd}_{\text {MaxPrt-1}}\right)$, where $\left[\operatorname{MaxRnd}_{k-1}\right.$, MaxRnd $_{k}$ ) is used only for the $k$ th priority. For example, suppose that there are six listening/signaling periods and that nodes with the highest priority may use the first three listening/signaling periods, $[0,2]$, and nodes with the lowest priority may use the last three, $[3,5]$. In the case of two neighbors with different listening period parameters, say, $[0,2]$ and $[3,5]$, that attempt to acquire the same broadcasting timeslot, the highest priority node always attempts to broadcast before the lowest priority one.

7.2. TDMA-Based Backoff. Let us consider two backoff parameters, $\mathrm{CW}_{\text {start }}$ and $\mathrm{CW}_{\text {end }}$, that refer to the maximal and minimal values of the contention window. Before selecting an unused timeslot, the procedure counts a random number of unused ones. Algorithm 2 presents an implementation of the select_unused() function that facilitates backoff strategies as an alternative to the implementation presented in line 29 of Algorithm 1.

The statically allocated variable count records the number of backoff steps that node $p_{i}$ takes until it reaches the zero value. Whenever the function select_unused() is invoked with count $_{i}=0$, node $p_{i}$ assigns to count ${ }_{i}$ a random integer from $\left[\mathrm{CW}_{\text {start }}, \mathrm{CW}_{\text {end }}\right]$ (cf. line 7). Whenever the value of count $t_{i}$ is not greater than the number of unused timeslots, the returned timeslot is selected uniformly at random (cf. lines 8 to 9). Otherwise, a $\perp$-value is returned after deducting the number of unused timeslots during the previous broadcasting round (cf. lines 6 and 10).

\section{Discussion}

Thus far, both schedule-based and nonschedule-based MAC algorithms could not consider timing requirements within a provably short recovery period that follows (arbitrary) 


\section{Additional constants and variables}

(2) $C W_{\text {start }}$ and $C W_{\text {end }}$ : backoff parameters

(4) count: statically allocated variable that counts the backoff steps

Function select_unused(set)

(6) let $r t n_{-} v a l=\perp_{-} v_{-} / /$indicate busy channel (default return value) if count $\leq 0$ then count $\leftarrow$ uniform_select $\left(\left[C W_{\text {start }}, C W_{\text {end }}\right]\right)$

(8) count $\leftarrow$ count $-\mid$ set $\mid$ if count $\leq 0$ then (count, rtn_val $) \leftarrow(0$, uniform_select $($ set $))$

(10) return $r$ tn_val

Algorithm 2: Select_unused() with TDMA-based backoff.

transient faults and network topology changes. This work proposes the first self-stabilizing TDMA algorithm for DynWANs that has a provably short convergence period. Thus, the proposed algorithm possesses a greater predictability degree, whilst maintaining low communication delays and high throughput.

In this discussion, we would like to point out the algorithm's ability to facilitate the satisfaction of severe timing requirements for DynWANs by numerically validating Theorem 7. As a case study, we show that, for the considered settings of Figure 2, the global convergence time is brief and definitive. Figure 3 shows that when allowing merely a small fraction of the bandwidth to be spent on frame control information, say, three listening/signaling periods, and when considering $99 \%$ probability to convergence within a couple of dozen TDMA frames, the proposed algorithm demonstrates a low dependency degree on the number of nodes in the network even when considering 10,000 nodes.

We have implemented the proposed algorithm, extensively validated our analysis via computer simulation, and tested it on a platform with more than two dozen nodes [18]. These results indeed validate that the proposed algorithm can indeed facilitate the implementation of MAC protocols that guarantee satisfying these severe timing requirements.

The costs associated with predictable communications, say, using cellular base stations, motivate the adoption of new networking technologies, such as MANETs and VANETs. In the context of these technologies, we expect that the proposed algorithm will contribute to the development of MAC protocols with a higher predictability degree.

\section{Appendices}

The proof of Theorem 2 uses the propositions in Appendices $\mathrm{A}$ and $\mathrm{B}$.

\section{A. Properties (1) to (3)}

Propositions A.1, A.2 and A.3 imply that properties (1), (2), and, respectively, (3) hold within two broadcasting rounds (or one complete broadcasting round). Let $R$ be an execution of the MAC algorithm in Algorithm $1, x>0$ an integer, and $c_{0}^{\text {timeslot }}(x)$ the first configuration in a complete broadcasting round $R(x)=\left(c_{0}^{\text {timeslot }}(x), \ldots c_{T-1}^{\text {carrier_sense } / \text { receive }}(x)\right)$. We note that $c_{0}^{\text {timeslot }}(x)$ follows an arbitrary starting configuration.

Proposition A.1 shows that, within a broadcasting round from $c_{0}^{\text {timeslot }}(x)$, Property (1) holds.

Proposition A.1. In $c_{0}^{\text {timeslot }}(x+1)$, it holds that signal $i=$ false.

Proof. The value of signal $i$ is updated in line 18 (assigned to true) and in lines 12, 20, and 27 (assigned to false). Let us look into these assignments.

In every timeslot, the value false is assigned to $\operatorname{signal}_{i}$ (cf. line 12). Suppose that the function send() is called, and thus, true is assigned to $\operatorname{signal}_{i}$ (line 18). We propose that before returning from the function send() and after true is assigned to $\operatorname{signal}_{i}$ (line 18), node $p_{i}$ must assign false to signal $i$ either in line 20 or 27 . To see that, let us look at lines 18 and 19. Eventually either signal $_{i}=$ false (because of an assignment in line 27) or $\rho(k)=$ true (line 19) holds (note the condition

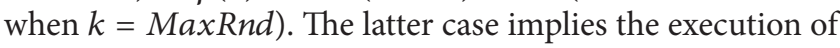
line 20 .

Proposition A.2 shows that, within a broadcasting round from $c_{0}^{\text {timeslot }}(x)$, Property (2) holds.

Proposition A.2. $\left(\exists t \in\right.$ unused_set $\left._{i} \backslash\left\{s_{i}\right\}\right) \leftrightarrow\left(\nexists p_{k} \in \mathcal{N}_{i}\right.$ : $\left.s_{k}=t\right)$ in $c_{0}^{\text {timeslot }}(x+1)$.

Proof. Recall that unused_set ${ }_{i}=\left\{k: \operatorname{unused}_{i}[k]=\operatorname{true}\right\}$ (see line 6) and that the proposition statement does not consider the cases in which: (1) $s_{i}=s_{k}$ (because $t \neq s_{i}$ ) in $c_{0}^{\text {timeslot }}(x+1)$, or (2) There exists a configuration $c \in R(x)$, such that $s_{k} \neq \perp$ in $c$ and $s_{k}=\perp$ in $c_{0}^{\text {timeslot }}(x+1)$ (because by unused_set's definition, $\perp$ is never in unused_set ${ }_{i}$ ).

We note that in every broadcasting round, node $p_{k} \in P$ at most once (1) Allocates the broadcasting timeslot $s_{k}$ (when $t_{k}=0$; see line 11), (2) transmits a packet (when $t_{k}=s_{k}$; see line 13), and (3) deallocates the broadcasting timeslot $s_{k}$ (by assigning $\perp$ to $s_{k}$ when $t_{k}=s_{k}$ and the carrier_sense $(t)$ event is raised; see line 26). Moreover, node $p_{i}$ updates unused ${ }_{i}[t]$ only in lines 12 (true) and 27 (false), when $p_{i}$ removes stale information just before timeslot $t$, and respectively, when the event carrier_sense $(t)$ is raised.

Line 12 is executed at the start of every timeslot, whereas line 27 is executed after and only when the event 
Defining optimal transmission probabilities for any choices of $T, n$, and $d_{i}$ is not possible. We choose to consider and look for optimal choices when $d_{i} \simeq T$ (the "hard" case) and make a case for a uniform probability $\rho_{i}=1 / n: i \in[1, n]$. Let us consider node $p_{i} \in P$ that competes, together with $k-1$ other neighbors, for the same unique timeslot. The probability that node $p_{i}$ wins the (listening/ signaling) competition is $\rho_{1}\left(1-\rho_{1}\right)^{k-1}$, where $\rho_{1}$ is the probability of choosing the first listening/signaling period. The value $\rho_{1}=1 / k$ maximizes this probability. In the more general case where there is more than one timeslot, we consider a strategy that aims at guessing the number, $k$, of competing neighbors, which the optimal probability of transmission depends on. During the first listening/signaling period, the strategy considers the case in which there are $n=$ MaxRnd signaling nodes, and thus, the transmission probability is $1 / \operatorname{Max} R n d$, where MaxRnd $\simeq T$. During the second listening/signaling period, the strategy considers the case in which there are MaxRnd - 1 neighbors, and thus, the transmission probability is $1 /(\operatorname{MaxR} n d-1)$, and so on. This sequential selection of the listening/signaling period leads to a uniform choice of a listening/signaling neighbor. The above strategy is driven by a heuristic in which nodes signal with probability that is optimal for the case of $n \simeq T$, and thus, it depends on the number of competing neighbors.

Box 2: Transition probability, $\rho_{i}$, for listening/signaling periods (line (19) in Algorithm 1).

carrier_sense $(t)$ is raised. The event carrier_sense $(t)$ is raised after and only when the node $p_{k} \in \mathcal{N}_{i}$ transmits in timeslot $t$. In other words, none of $p_{i}$ 's neighbors, $p_{k} \in \mathcal{N}_{i}$, that transmits in timeslot $s_{k}=t$, can avoid causing the event carrier_sense $(t)$ to be raised, and timeslot $t$ to be included in unused_set $\backslash \backslash\left\{s_{i}\right\}$.

Proposition A.3 shows that, within a broadcasting round from $c_{0}^{\text {timeslot }}(x)$, Property (3) holds.

Proposition A.3. $\left(s_{i} \neq \perp\right) \vee\left(\right.$ unused_set $\left._{i} \backslash\left\{s_{i}\right\} \neq \emptyset\right)$ holds in $c_{0}^{\text {timeslot }}(x+1)$.

Proof. If $s_{i} \neq \perp$ in $c_{0}^{\text {timeslot }}(x+1)$, we are done. Let us suppose that $s_{i}=\perp$ in $c_{0}^{\text {timeslot }}(x+1)$ and show that unused_set $\backslash \backslash\left\{s_{i}\right\} \neq \emptyset$ in $c_{0}^{\text {timeslot }}(x+1)$.

Let us assume, in the way of proof by contradiction, that, unused_set ${ }_{i} \backslash\left\{s_{i}\right\}=\emptyset$ and show that $d_{i} / T>1$, that is, a contradiction with the assumption that for all $p_{i} \in P: d_{i} / T \lessgtr$ 1 .

Recall that unused_set ${ }_{i}=\left\{k:\right.$ unused $_{i}[k]=$ true $\} \subseteq$ $[0, T-1]$ (see line 6). Therefore, the assumption that $s_{i}=\perp$ implies that unused_set ${ }_{i}=$ unused_set $_{i} \backslash\left\{s_{i}\right\} \subseteq[0, T-1]$, because by unused_set's definition, $\perp$ is never in unused_set $i$.

By Proposition A.2, we can say that for all $t \in[0, T-$ 1]: ( $\nexists t \in$ unused_set $\left.{ }_{i}\right) \leftrightarrow\left(\exists p_{k} \in \mathcal{N}_{i}: s_{k}=t\right)$. Since unused_set ${ }_{i} \subseteq[0, T-1]$, we can write $[0, T-1] \backslash$ unused_set $_{i} \subseteq$ $\left\{s_{k} \in[0, T-1]: p_{k} \in \mathcal{N}_{i}\right\}$. By the fact that unused_set ${ }_{i}=\emptyset$, we have that $T \leq\left|\left\{s_{k} \in[0, T-1]: p_{k} \in \mathcal{N}_{i}\right\}\right|$. Since $d_{i}=\left|\mathcal{N}_{i}\right|$ (by definition), we have that $\left|\left\{s_{k} \in[0, T-1]: p_{k} \in \mathcal{N}_{i}\right\}\right| \leq d_{i}$, which implies $T \leq d_{i}$ : a contradiction with the assumption that $d_{i} / T \lesseqgtr 1$.

\section{B. Properties (4) to (5)}

Appendix A shows that, starting from an arbitrary configuration, node $p_{i} \in P$ enters the relative state Ready within two broadcasting rounds. This section considers the probability for $p_{i}$ to enter the relative states Obtaining and Allocated.

Let $x>0$ and $R$ be an execution of the MAC algorithm in Algorithm 1. Suppose that $c_{0}^{\text {timeslot }}(x)$ is the first configuration in a complete broadcasting round $R(x)$ for which properties (1) to (3) hold in configuration $c_{0}^{\text {timeslot }}(x)$ with respect to node $p_{i} \in P$; that is, $p_{i}$ is in relative state Ready, Obtaining or Allocated. Propositions B.1, B.2 and B.3 show that there is a nonzero probability that node $p_{i}$ enters the relative state Allocated from either Ready or Obtaining in configuration $c_{0}^{\text {timeslot }}(x+1)$.

Proposition B.1 shows that $p_{i}$ attempts to broadcast once in every round.

Proposition B.1. During broadcasting round $R(x), p_{i}$ executes line 13 and calls the function send().

Proof. If $s_{i} \neq \perp$ in $c_{0}^{\text {timeslot }}(x)$, we are done by lines 11 and 13 . Let us consider the case of $s_{i}=\perp$ in $c_{0}^{\text {timeslot }}(x)$. By Property (4), unused_set $\neq \emptyset \emptyset$, and thus, when line 11 is executed, the function select_unused() returns a non- $\perp$ element from unused_set $_{i}$ and $s_{i} \neq \perp$ when executing line 13 .

Propositions B.2 and B.3 consider the set $M_{i}(x+1)=$ $\left\{p_{k} \in \mathcal{N}_{i}: s_{k}=t^{\prime}\right\}$ and the number $m_{i}=\left|M_{i}(x+1)\right|$ of $p_{i}$ 's neighbors that attempt to broadcast during $p_{i}$ 's timeslot, $t^{\prime}$, of broadcasting round $R(x)$.

Let $\rho_{j}$ be the probability for $p_{i}$ to transmit in the $j$ th listening/signaling period of timeslot $t^{\prime}$ (cf. line 19). This paper considers the concrete transmission probability $\rho_{i}=$ $1 /$ MaxRnd. We motivate our implementation choice of the transmission probability, $\rho_{i}$, in Box 2 . Note that the sequential selection of the broadcasting rounds with probability $1 /(\operatorname{MaxR}$ nd $-k+1)$ leads to the uniform selection $\rho_{k}=$ $1 / \operatorname{Max}$ Rnd. 
Proposition B. 2 considers $p_{i}$ 's chances to be the only one to transmit in its neighborhood.

Proposition B.2. There is a nonzero probability, OnlyOne ${ }_{i}(x)$ (cf. (B.1)), that only node $p_{i}$ transmits in its broadcasting timeslot, $t^{\prime}$, of broadcasting round $R(x)$.

One has

$$
\begin{aligned}
\left.\operatorname{OnlyOne}_{i}(x)\right|_{m_{i}>0}= & \rho_{1}\left(1-\rho_{1}\right)^{m_{i}}+\rho_{2}\left(1-\rho_{1}-\rho_{2}\right)^{m_{i}} \\
& +\cdots+\rho_{n-1}\left(1-\sum_{\ell=1}^{n-1} \rho_{k}\right)^{m_{i}}
\end{aligned}
$$

Proof. We show that there is a nonzero probability that only node $p_{i}$ transmits in its broadcasting timeslot, $t^{\prime}$, of broadcasting round $R(x)$. Let us look at $p_{i}$ and the nodes in $M_{i}(x)$ while they attempt to broadcast in the steps $a_{i}^{\text {timeslot } t^{\prime}}(x)$ and $a_{k}^{\text {timeslot, } t^{\prime}}(x)_{\mid k \in M_{i}(x)}$. All of these steps include the execution of line 19; namely, each node chooses to transmit in listening/signaling period $\ell \in[0, \operatorname{Max} R n d]$ with probability $\rho_{\ell}=$ $1 /(\operatorname{Max} R n d-\ell)$. Therefore, for any MaxRnd > 0 , there is a nonzero probability, OnlyOne $e_{i}(x)$, that, during timeslot $t^{\prime}$, node $p_{i}$ transmits in the listening/signaling period $a \in$ MaxRnd and no node in $M_{i}(x)$ transmits in round $a$ (or in an earlier one).

We note that the fact that $p_{i}$ transmits first during timeslot $t^{\prime}$ implies that it is the only one to transmit during $t^{\prime}$. This is because once $p_{i}$ transmits a beacon in step $a_{i}^{\text {timeslot, } t^{\prime}}(x)$ (which includes the execution of line 21), node $p_{j} \in \mathcal{N}_{i} \supseteq M_{i}(x)$ raises the event carrier_sense $\left(t^{\prime}\right)$ immediately after $a_{i}^{\text {timeslot, } t^{\prime}}(x)$. Thus, for all $p_{j} \in M_{i}(x)$ we have that immediately after step $a_{i}^{\text {timeslot, } t^{\prime}}(x)$, node $p_{j}$ takes step $a_{j}^{\text {carrier_sense, } t^{\prime}}(x)$, which includes the execution of lines 26 and 27 that assign $\perp$ to $s_{j}$ and false to signal ${ }_{j}$. Thus, $p_{j}$ leaves the (listening/signaling) competition for timeslot $t^{\prime}$ (see line 18 ) and does not transmit its DATA packet (see line 23).

We now turn to calculate OnlyOne $e_{i}(x)$. Let the variable $m_{i}=\left|M_{i}(x)\right|$ denote the number of nodes that select the

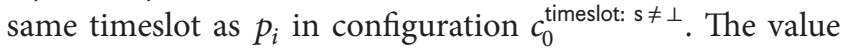
of OnlyOne $e_{i}(x)$ depends on the value of $m_{i}$, and we denote this dependence with the notation $\left.q(i)\right|_{m_{i}}$ (conditional probability). It means the value of OnlyOne ${ }_{i}(x)$ depends on the value of $m_{i}$. The value of OnlyOne $e_{i}(x)$ for $m_{i}=0$ is OnlyOne $\left._{i}(x)\right|_{m_{i}=0}=1$. For the case of $m_{i}>0$, OnlyOne $e_{i}(x)$ 's value is given by (B.1) (that appears again next), where $\rho_{j}$ is the probability for transmitting in the $j$ th listening/signaling period.

$$
\begin{aligned}
& \text { Consider } \\
& \begin{aligned}
\text { OnlyOne }\left._{i}(x)\right|_{m_{i}>0} \\
=\rho_{1}\left(1-\rho_{1}\right)^{m_{i}}+\rho_{2}\left(1-\rho_{1}-\rho_{2}\right)^{m_{i}} \\
\quad+\cdots+\rho_{n-1}\left(1-\sum_{\ell=1}^{n-1} \rho_{k}\right)^{m_{i}}[\text { clone of }(\mathrm{B} .1)]
\end{aligned}
\end{aligned}
$$

We note that the $j$ th term in (B.1) is the probability that node $p_{i}$ selects the $j$ th listening/signaling period and all its neighbors select a later listening/signaling period.
Proposition B.3 shows that once a node is the only one in its neighborhood to transmit during its broadcasting timeslot, it enters the relative state Allocated.

Proposition B.3. $M_{i}(x)=\emptyset$ (or having none of the nodes in $M_{i}(x)$ transmitting during timeslot $\left.t^{\prime}\right)$ implies that node $p_{i}$ is in the relative state Allocated in $c_{0}^{\text {timeslot }}(x+1)$.

Proof. We need to show that, in $c_{0}^{\text {timeslot }}(x+1)$, we have that $s_{i}=t^{\prime} \neq \perp$ and for all $p_{j} \in \mathcal{N}_{i}: s_{i} \neq s_{j}$.

Showing That $s_{i}=t^{\prime} \neq \perp$ in $c_{0}^{\text {timeslot }}(x+1)$. The proposition assumes that $t^{\prime} \neq \perp$ in $c_{0}^{\text {timeslot }}(x)$. We wish to show that $s_{i}=t^{\prime}$ in $c_{0}^{\text {timeslot }}(x+1)$, which implies that $s_{i} \neq \perp$ holds in $c_{0}^{\text {timeslot }}(x+1)$ and throughout $R(x+1)$.

Since the variable $s_{i}$ is assigned only in lines 11 (when $t_{i}=$ 0 ) and 26 (when $t_{i}=t^{\prime}$ ), it is sufficient to show that line 26 is not executed by any step during timeslot $t^{\prime}$ of broadcasting round $R(x)$, that is, $a_{i}^{\text {carrier_sense, } t^{\prime}}(x) \notin R(x)$.

Node $p_{i}$ raises the event carrier_sense only during timeslots in which $p_{i}$ 's neighbor, $p_{j}$, transmits. By the proposition assumptions that, during timeslot $t^{\prime}$ of broadcasting round $R(x)$, none of $p_{i}$ 's neighbors transmits, we have $a_{i}^{\text {carrier_sense, } t^{\prime}}(x) \notin R(x)$. Moreover, $a_{i}^{\text {timeslot, } t^{\prime}}(x+1)$ does not include an execution of line 11 that changes the value of $s_{i}$, because $s_{i}=t^{\prime} \neq \perp$ in $c_{0}^{\text {timeslot }}(x+1)$.

Showing That for All $p_{j} \in \mathcal{N}_{i}: s_{i} \neq s_{j}$ in $c_{0}^{\text {timeslot }}(x+1)$. The proposition assumes that for all $p_{j} \in \mathcal{N}_{i}: s_{i} \neq s_{j}$ in $c_{0}^{\text {timeslot }}(x)$. We wish to show that the same holds in $c_{0}^{\text {timeslot }}(x+1)$. Since the variable $s_{j}$ is assigned to a non- $\perp$ value only in line 11 when $t_{i}=0$, it is sufficient to show that when line 11 is executed in step $a_{j}^{\text {timeslot, } 0}(x+1)$ the function select_unused() considers a set that does not include $p_{i}$ 's timeslot, $s_{i}$. This is implied by the facts that for all $p_{j} \in \mathcal{N}_{i}$ : unused $_{j}\left[t^{\prime}\right]=$ false (Claim 10.1) and $s_{i}=t^{\prime}$ (first item of (II) of this proof) in $c_{0}^{\text {timeslot }}(x+1)$.

\section{Theorem 2}

Theorem 2 shows that all nodes are allocated eventually with timeslots (convergence) and once all nodes are allocated, they stay this way (closure).

Theorem 2 (self-stabilization). The MAC algorithm in Algorithm 1 is a self-stabilizing algorithm with respect to the task $\tau_{\text {TDMA }}$.

Proof. After the previous proof of propositions, we can demonstrate this theorem.

(i) Convergence. We need to show that properties (1) to (5) eventually hold in configuration $c_{0}^{\text {timeslot }}(x+y)$ for a finite value of $y>0$. Propositions A.1, A.2 and A.3 imply that properties (1), (2), and, respectively, (3) within two broadcasting round.

Propositions B.1, B.2 and B.3 show that there is a nonzero probability that node $p_{i}$ enters the rela- 
tive state Allocated from either Ready or Obtaining within one broadcasting round. Thus, by analyzing the expected time of the scheduler-luck games [4, 51], we have that $y$ has a finite value. Further analysis of $y$ appears in Theorems 3 and 7.

(ii) Closure. Suppose that $c_{0}^{\text {timeslot }}(x) \in R$ is a safe configuration and let $p_{i} \in P$ be any node. By the assumption that $c_{0}^{\text {timeslot }}(x)$, we have that $p_{i}$ is in the relative state Allocated; that is, properties (1) to (5) hold for any node $p_{i}$. We need to show that properties (1) to (5) hold in configuration $c_{0}^{\text {timeslot }}(x+1)$.

Propositions A.1, A.2, and A.3 imply properties (1), (2), and respectively, (3) (within one complete broadcasting round).

Properties (4) to (5) are implied by Proposition B.3 and the fact that Properties (4) to (5) hold in $c_{0}^{\text {timeslot }}(x)$, that is, $M(x)=\emptyset$.

\section{Bounding OnlyOne $e_{i}(x)$}

Propositions 5 and D.2 bound OnlyOne $(x)$ 's value, where $R(x)$ is the $x$ th broadcasting round in execution $R$ of the MAC algorithm in Algorithm 1. We assume that properties (1) to (5) hold in the first configuration, $c_{0}^{\text {timeslot }}(x)$, of $R(x)$. These bounds are obtained by computing the expectation of $\left.q_{i}\right|_{m_{i}}$ with respect to $m_{i}$, where $M_{i}(x)=\left\{p_{k} \in \mathcal{N}_{i}: s_{k}=t^{\prime}\right\}$ in $c_{0}^{\text {timeslot }}(x)$ and $m_{i}=\left|M_{i}(x)\right|$. The reason is that $m_{i}$ is a random variable, that is, $q_{i}=E\left(\left.O n l y O n e_{i}(x)\right|_{m_{i}}\right)$, where the expectation is computed with respect to the random variable $m_{i}$.

We note that all the terms in (B.1) are convex functions of $m_{i}$. This means that by Jensen's inequality, we obtain a lower bound of $q_{i}$ in (D.1) by evaluating the expression $\left.q_{i}\right|_{m_{i}}$ at $m_{i}$ 's expectation, $E\left(m_{i}\right)$ [52].

One has

$$
q_{i}=E\left(\left.q_{i}\right|_{m_{i}}\right) \geq\left. q_{i}\right|_{E\left(m_{i}\right)}
$$

The expression on the right side of the inequality can be again lower bounded if we estimate an upper bound for $E\left(m_{i}\right)$. We proceed to the computations in the proof of Proposition D.2 after demonstrating Proposition D.1 which shows that $E\left(m_{i}\right)$ is bounded by the ratio $d_{i} / T$, which is rather intuitive but needs to be proved.

Proposition D.1. In configuration $c_{0}^{\text {timeslot }}(x)$ it holds that $E\left(m_{i}\right) \leq d_{i} / T$, where $m_{i}=\left|M_{i}(x)\right|$.

Proof. We show that $E\left(m_{i}\right)=d_{i} / T$ by considering configuration $c_{0}^{\text {timeslot }}(x)$. The maximal number of $p_{i}$ 's neighbors that might choose the same timeslot as $p_{i}$ in configuration $c_{0}^{\text {timeslot }}(x)$ is $\sum_{p_{j} \in \mathcal{N}_{i}} 1_{\left\{s_{j}=\perp\right\}}$, because any node, $p_{j} \in \mathcal{N}_{i}$, that chooses a new broadcasting timeslot immediately before $c_{0}^{\text {timeslot }}(x)$ must have $s_{j}=\perp$ in configuration $c_{0}^{\text {timeslot }}(x)$. We compute the expected value of $m_{i}$ in (D.2) as a function of the number of empty timeslots, $e_{i}$, that $p_{i}$ selects from when choosing a new broadcasting timeslot, where $e_{i}=$ $\mid$ unused_set ${ }_{i} \mid$ in configuration $c_{0}^{\text {timeslot }}(x)$.
Consider

$$
\begin{aligned}
E\left(m_{i}\right) & =\sum_{t \in E_{i}} E\left(m_{i} \mid s_{i}=t\right) \operatorname{Pr}\left(s_{i}=t\right) \\
& =\sum_{t \in E_{i}} \frac{1}{e_{i}} E\left(m_{i} \mid s_{i}=t\right) \\
& =\sum_{t \in E_{i}} \frac{1}{e_{i}} E\left(\sum_{p_{j} \in \mathcal{N}_{i}} 1_{\left\{p_{j} \text { chooses timeslot } t\right\}} \mid s_{i}=t\right) \\
& =\sum_{t \in E_{i}} \frac{1}{e_{i}} \sum_{p_{j} \in \mathcal{N}_{i}} \frac{1}{\left|E_{j}\right|} 1_{\left\{t \in E_{j}\right\}} 1_{\left\{s_{j}=\perp\right\}} .
\end{aligned}
$$

Our assumption that $d_{i} \leq T-1$ implies that $e_{i}>0$. Using that $d_{i}=\sum_{p_{j} \in \mathcal{N}_{i}}\left(1_{\left\{s_{j} \neq \perp\right\}}+1_{\left\{s_{j}=\perp\right\}}\right)$ and, $e_{i} \geq T-\sum_{p_{j} \in \mathcal{N}_{i}} 1_{\left\{s_{j} \neq \perp\right\}}$, we obtain the following:

$$
\begin{aligned}
E\left(m_{i}\right) & \leq \sum_{t \in E_{i}} \frac{1}{T-d_{i}+\sum_{p_{j} \in \mathcal{N}_{i}} 1_{\left\{s_{j}=\perp\right\}}} \sum_{p_{j} \in \mathcal{N}_{i}} \frac{1_{\left\{t \in E_{j}\right\}} 1_{\left\{s_{j}=\perp\right\}}}{\left|E_{j}\right|} \\
& =\frac{1}{T-d_{i}+\sum_{p_{j} \in \mathcal{N}_{i}} 1_{\left\{s_{j}=\perp\right\}}} \sum_{p_{j} \in \mathcal{N}_{i}} \frac{1_{\left\{s_{j}=\perp\right\}}}{\left|E_{j}\right|} \underbrace{\sum_{t \in E_{i}} 1_{\left\{t \in E_{j}\right\}}}_{\left|E_{i} \cap E_{j}\right|} \\
& \leq \frac{\sum_{p_{j} \in \mathcal{N}_{i}} 1_{\left\{s_{j}=\perp\right\}}}{T-d_{i}+\sum_{p_{j} \in \mathcal{N}_{i}} 1_{\left\{s_{j}=\perp\right\}}} \leq \frac{d_{i}}{T} .
\end{aligned}
$$

Proposition D.2. One has

$$
q_{i} \geq \sum_{k=1}^{n} \rho_{k}\left(1-\sum_{\ell=1}^{k} \rho_{k}\right)^{d_{i} / T} \quad[\text { clone of }(6)]
$$

Proof. Proposition D.1 shows that $E\left(m_{i}\right) \leq d_{i} / T$. The proposition is demonstrated by evaluating expression (B.1) at $E\left(m_{i}\right)=d_{i} / T$; see (D.1).

Proposition 5 considers the concrete transmission probability $\rho_{i}=1 / \operatorname{MaxR} n d$.

Proposition 5. Let $\rho_{i}=1 /$ MaxRnd. Equation (16) bounds from below the probability $q_{i}$.

Proof. In this proof, we use the letter $n$ instead of MaxRnd for reason of space. We replace $\rho_{i}$ with $1 / n$ in (6) to obtain (D.5).

Consider

$$
q_{i} \geq \sum_{k=1}^{n} \frac{1}{n}\left(1-\frac{k}{n}\right)^{d_{i} / T}
$$

Equation (D.6) is more compact than (D.5) and it is obtained by the fact that the function $(1-x)^{s}$ is convex. 


$$
\begin{aligned}
& \text { Consider } \\
& \begin{aligned}
q_{i} & \geq \sum_{k=1}^{n} \frac{1}{n}\left(1-\frac{k}{n}\right)^{d_{i} / T} \\
& =\frac{1}{2 n} \sum_{k=1}^{n}\left[\left(1-\frac{k}{n}\right)^{d_{i} / T}+\left(1-\frac{n-k+1}{n}\right)^{d_{i} / T}\right] \\
& \geq(\text { convexity }) \frac{1}{n} \sum_{k=1}^{n}\left(1-\frac{n+1}{2 n}\right)^{d_{i} / T}=\left(1-\frac{n+1}{2 n}\right)^{d_{i} / T} .
\end{aligned}
\end{aligned}
$$

Another way to bound (D.5) is by considering the decreasing function $y \rightarrow(1-y)^{x}$, as in the following:

$$
\begin{aligned}
q_{i} & \geq \sum_{k=1}^{n} \frac{1}{n}\left(1-\frac{j}{n}\right)^{d_{i} / T} \\
& \geq \int_{1 / n}^{1}(1-y)^{d_{i} / T} d y=\frac{1}{d_{i} / T+1}\left(1-\frac{1}{n}\right)^{d_{i} / T+1} .
\end{aligned}
$$

\section{Acknowledgments}

The authors thank Thomas Petig and Oscar Morales for helping with improving the presentation. The work of Elad M. Schiller was partially supported by the EC, through project FP7-STREP-288195, KARYON (kernel-based architecture for safety-critical control). The proceeding and technical versions of this work appears in [53] and, respectively, [54].

\section{References}

[1] H. Hartenstein and K. Laberteaux, VANET: Vehicular Applications and Inter-Networking Technologies, Wiley, 2010.

[2] K. Bilstrup, E. Uhlemann, E. G. Ström, and U. Bilstrup, "Evaluation of the IEEE 802.11p MAC method for vehicle-tocehicle communication," in Proceedings of the 68th Semi-Annual IEEE Vehicular Technology (VTC '08), pp. 1-5, September 2008.

[3] K. Bilstrup, E. Uhlemann, E. G. Ström, and U. Bilstrup, "On the ability of the 802.11p MAC method and STDMA to support real-time vehicle-to-vehicle communication," Eurasip Journal on Wireless Communications and Networking, vol. 2009, Article ID 902414, 2009.

[4] S. Dolev, Self-Stabilization, MIT Press, 2000.

[5] S. Dolev and T. Herman, "Superstabilizing protocols for dynamic distributed systems," Chicago Journal of Theoretical Computer Science, 1997.

[6] N. Abramson, "Development of the ALOHANET," IEEE Transactions on Information Theory, vol. 31, no. 2, pp. 119-123, 1985.

[7] W. G. Schmidt, "Satellite time-division multiple access systems: past, present and future," Telecommunications, vol. 7, no. 8, pp. 21-24, 1973.

[8] M. Haenggi, "Outage, local throughput, and capacity of random wireless networks," IEEE Transactions on Wireless Communications, vol. 8, no. 8, pp. 4350-4359, 2009.

[9] P. Leone, M. Papatriantafilou, and E. Michael Schiller, "Relocation analysis of stabilizing MAC algorithms for large-scale mobile ad hoc networks," in Proceedings of the 5th International Workshop on Algorithm Aspects of Wireless Sensor Networks (ALGOSENSORS '09), pp. 203-217, 2009.
[10] T. Herman and S. Tixeuil, "A distributed TDMA slot assignment algorithm for wireless sensor networks," in Proceedings of the 5th International Workshop on Algorithmic Aspects of Wireless Sensor Networks (ALGOSENSORS '04), vol. 3121 of Lecture Notes in Computer Science, pp. 45-58, Springer, 2004.

[11] W. Ye, J. Heidemann, and D. Estrin, "An energy-efficient MAC protocol for wireless sensor networks," in Proceedings of the IEEE INFOCOM, pp. 1567-1576, June 2002.

[12] A. Cornejo and F. Kuhn, "Deploying wireless networks with beeps," in Proceedings of the 24th International Symposium on Distributed Computing (DISC '10), N. A. Lynch and A. A. Shvartsman, Eds., vol. 6343 of Lecture Notes in Computer Science, pp. 148-162, Springer, 2010.

[13] R. Rom and F. A. Tobagi, "Message-based priority functions in local multiaccess communication systems," Computer Networks, vol. 5, no. 4, pp. 273-286, 1981.

[14] R. Scopigno and H. A. Cozzetti, "Mobile slotted Aloha for Vanets," in Proceedings of the IEEE 70th Vehicular Technology Conference Fall (VTC '09), pp. 1-5, September 2009.

[15] F. Yu and S. Biswas, "Self-configuring TDMA protocols for enhancing vehicle safety with DSRC based vehicle-to-vehicle communications," IEEE Journal on Selected Areas in Communications, vol. 25, no. 8, pp. 1526-1537, 2007.

[16] S. Viqar and J. L. Welch, "Deterministic collision free communication despite continuous motion," in Proceedings of the 5th International Workshop on Algorithm Aspects of Wireless Sensor Networks (ALGOSENSORS '09), pp. 218-229, 2009.

[17] R. Scopigno and H. A. Cozzetti, "GNSS synchronization in Vanets," in Proceedings of the 3rd International Conference on New Technologies, Mobility and Security (NTMS '09), pp. 1-5, December 2009.

[18] M. Mustafa, M. Papatriantafilou, E. Michael Schiller, A. Tohidi, and P. Tsigas, "Autonomous TDMA alignment for VANETs," in Proceedings of the IEEE 76th Vehicular Technology Conference (VTC '12), 2012.

[19] A. Daliot, D. Dolev, and H. Parnas, "Self-stabilizing pulse synchronization inspired by biological pacemaker networks," in Proceedings of the 6th International Symposium on SelfStabilizing Systems (SSS '03), S.-T. Huang and T. Herman, Eds., vol. 2704 of Lecture Notes in Computer Science, pp. 32-48, Springer, 2003.

[20] Y. Tadokoro, K. Ito, J. Imai, N. Suzuki, and N. Itoh, "Advanced transmission cycle control scheme for autonomous decentralized TDMA protocol in safe driving support systems," in Proceedings of the IEEE Intelligent Vehicles Symposium, pp. 10621067, June 2008.

[21] H. A. Cozzetti and R. Scopigno, "RR-Aloha+: a slotted and distributed MAC protocol for vehicular communications," in Proceedings of the IEEE Vehicular Networking Conference (VNC '09), pp. 1-8, October 2009.

[22] M. Lenoble, K. Ito, Y. Tadokoro, M. Takanashi, and K. Sanda, "Header reduction to increase the throughput in decentralized TDMA-based vehicular networks," in Proceedings of the IEEE Vehicular Networking Conference (VNC '09), pp. 1-4, October 2009.

[23] R. Scopigno and H. A. Cozzetti, "Evaluation of time-space efficiency in CSMA/CA and slotted vanets," in Proceedings of the IEEE 72nd Vehicular Technology Conference Fall (VTC '10), pp. 1-5, September 2010.

[24] F. Abrate, A. Vesco, and R. Scopigno, "An analytical packet error rate model for WAVE receivers," in Proceedings of the IEEE 74th 
Vehicular Technology Conference (VTC '11), pp. 1-5, September 2011.

[25] H. A. Cozzetti, R. M. Scopigno, L. Casone, and G. Barba, "Comparative analysis of IEEE 802.11p and MS-aloha in vanet scenarios," in Proceedings of the IEEE Asia-Pacific Services Computing Conference (APSCC '09), pp. 64-69, December 2009.

[26] M. Demirbas and M. Hussain, "A MAC layer protocol for priority-based reliable multicast in wireless ad hoc networks," in Proceedings of the 3rd International Conference on Broadband Communications, Networks and Systems (BROADNETS '06), October 2006.

[27] J. R. S. Blair and F. Manne, "An efficient self-stabilizing distance2 coloring algorithm," in Proceedings of the SIROCCO, S. Kutten and J. Zerovnik, Eds., vol. 5869 of Lecture Notes in Computer Science, pp. 237-251, Springer, 2009.

[28] S. Gollakota and D. Katabi, "Zigzag decoding: combating hidden terminals in wireless networks," in Proceedings of the ACM SIGCOMM Conference on Data Communication (SIGCOMM '08), V. Bahl, D. Wetherall, S. Savage, and I. Stoica, Eds., pp. 159170, August 2008.

[29] F. Kuhn, N. A. Lynch, and C. C. Newport, "The abstract MAC layer," in Proceedings of the 23rd International Symposium on Distributed Computing (DISC '09), I. Keidar, Ed., vol. 5805 of Lecture Notes in Computer Science, pp. 48-62, Springer, 2009.

[30] M. M. Halldórsson and R. Wattenhofer, "Wireless communication is in APX," in Proceedings of the ICALP, S. Albers, A. Marchetti-Spaccamela, Y. Matias, S. E. Nikoletseas, and W. Thomas, Eds., vol. 5555 of Lecture Notes in Computer Science, pp. 525-536, Springer, 2009.

[31] O. Goussevskaia, R. Wattenhofer, M. M. Halldórsson, and E. Welzl, "Capacity of arbitrary wireless networks," in Proceedings of the INFOCOM, pp. 1872-1880, IEEE, 2009.

[32] R. Wattenhofer, “Theory meets practice, it's about time!," in Proceedings of the 36th International Conference on Current Trends in Theory and Practice of Computer Science (SOFSEM '10), Špindlerův Mlýn, Czech Republic, January 2010.

[33] C. Lenzen, T. Locher, P. Sommer, and R. Wattenhofer, "Clock synchronization: open problems in theory and practice," in Proceedings of the 36th International Conference on Current Trends in Theory and Practice of Computer Science (SOFSEM '10), Špindlerův Mlýn, Czech Republic, January 2010.

[34] J. Schneider and R. Wattenhofer, "Coloring unstructured wireless multi-hop networks," in Proceedings of the ACM Symposium on Principles of Distributed Computing (PODC '09), S. Tirthapura and L. Alvisi, Eds., pp. 210-219, August 2009.

[35] A. Jhumka and S. S. Kulkarni, "On the design of mobilitytolerant TDMA-based media access control (MAC) protocol for mobile sensor networks," in Proceedings of the ICDCIT, T. Janowski and H. Mohanty, Eds., vol. 4882 of Lecture Notes in Computer Science, pp. 42-53, Springer, 2007.

[36] C. Lenzen, J. Suomela, and R. Wattenhofer, "Local algorithms: self-stabilization on speed," in Proceedings of the 11th International Symposium on Stabilization, Safety, and Security of Distributed Systems (SSS '09), R. Guerraoui and F. Petit, Eds., vol. 5873 of Lecture Notes in Computer Science, pp. 17-34, Springer, 2009.

[37] A. Lagemann, J. Nolte, C. Weyer, and V. Turau, "Mission statement: applying self-stabilization to wireless sensor networks," in Proceedings of the 8th GI/ITG KuVS Fachgespräch "Drahtlose Sensornetze” (FGSN '09), pp. 47-49, August 2009.
[38] M. Arumugam and S. S. Kulkarni, "Self-stabilizing deterministic TDMA for sensor networks," in Proceedings of the 2nd International Conference on Distributed Computing and Internet Technology (ICDCIT '05), G. Chakraborty, Ed., vol. 3816 of Lecture Notes in Computer Science, pp. 69-81, Springer, 2005.

[39] M. Arumugam and S. S. Kulkarni, "Self-stabilizing deterministic time division multiple access for sensor networks," Journal of Aerospace Computing, Information and Communication, vol. 3, no. 8, pp. 403-419, 2006.

[40] S. S. Kulkarni and M. Arumugam, "Infuse: a TDMA based data dissemination protocol for sensor networks," International Journal of Distributed Sensor Networks, vol. 2, no. 1, pp. 55-78, 2006.

[41] P. Leone, M. Papatriantafilou, E. Michael S, and G. Zhu, "Analyzing protocols for media access control in large-scale mobile ad hoc networks," in Proceedings of the Workshop on Self-Organising Wireless Sensor and Communication Networks (Somsed '09), Hamburg, Germany, October 2009.

[42] P. Leone, M. Papatriantafilou, E. Michael Schiller, and G. Zhu, "Chameleon-mac: adaptive and self-? algorithms for media access control in mobile ad hoc networks," in Proceedings of the12th International Symposium on Stabilization, Safety, and Security of Distributed Systems (SSS '10), S. Dolev, J. Arturo Cobb, M. J. Fischer, and M. and Yung, Eds., vol. 6366 of Lecture Notes in Computer Science, pp. 468-488, Springer, 2010.

[43] D. Aldous and J. Fill, "Reversible markov chain and random walks on graph," 1999, http://www.stat.berkeley.edu/ aldous/ RWG/book.html.

[44] B. Hofmann-Wellenhof, H. Lichtenegger, and J. Collins, Global Positioning System. Theory and Practice, Springer, 1993.

[45] K. Jamieson, B. Hull, A. Miu, and H. Balakrishnan, "Understanding the real-world performance of carrier sense," in Proceedings of the ACM SIGCOMM Workshop on Experimental Approaches to Wireless Network Design and Analysis (E-WIND '05), pp. 52-57, August 2005.

[46] R. Cogburn, "The ergodic theory of Markov chains in random environments," Zeitschrift für Wahrscheinlichkeitstheorie und Verwandte Gebiete, vol. 66, no. 1, pp. 109-128, 1984.

[47] S. M. Ross, Stochastic Processes, John Wiley \& Sons, 1996.

[48] J. M. Steele, The Cauchy-Schwartz Master Class, Cambridge University Press,, 2004.

[49] T. Lindvall, Lectures on the Coupling Method, Dover Publications, 1992.

[50] A. M. Crocker, W. L. Godson, and C. M. Penner, "Frontal contour charts," Journal of the Atmospheric Sciences, vol. 4, no. 3, 1947.

[51] S. Dolev, A. Israeli, and S. Moran, "Analyzing expected time by scheduler-luck games," IEEE Transactions on Software Engineering, vol. 21, no. 5, pp. 429-439, 1995.

[52] J. L. W. V. Jensen, "Sur les fonctions convexes et les inégalités entre les valeurs moyennes," Acta Mathematica, vol. 30, no. 1, pp. 175-193, 1906.

[53] P. Leone and E. Michael Schiller, "Self-stabilizing tdma algorithms for dynamic wireless ad-hoc networks," in Proceedings of the ALGOSENSORS, A. Bar-Noy and M. M. Halldórsson, Eds., Lecture Notes in Computer Science, Springer, 2012.

[54] P. Leone and E. M. Schiller, "Self-stabilizing TDMA algorithms for dynamic wireless ad-hoc networks," abs 1210.3061, submitted in CoRR, 2012, http://arxiv.org/abs/1210.3061. 


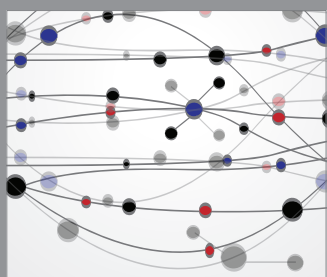

The Scientific World Journal
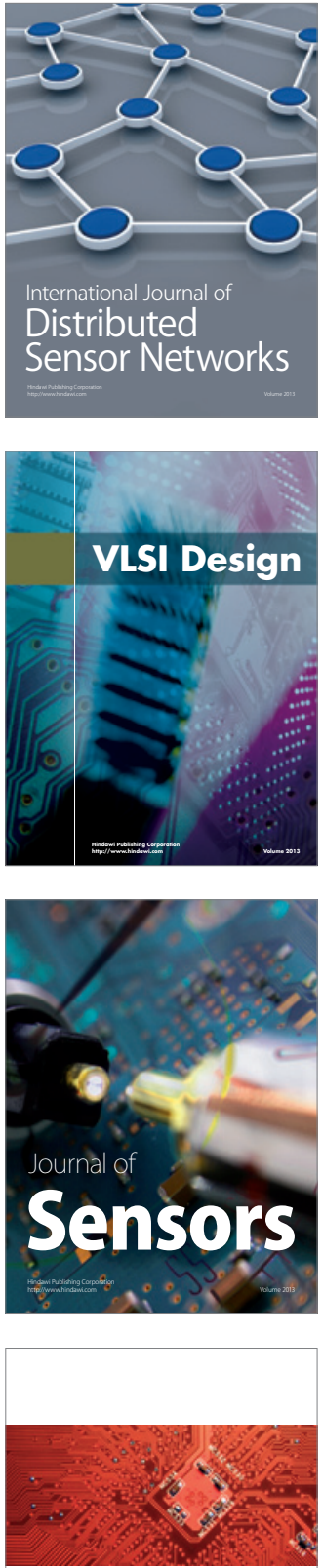

ISRN

Electronics
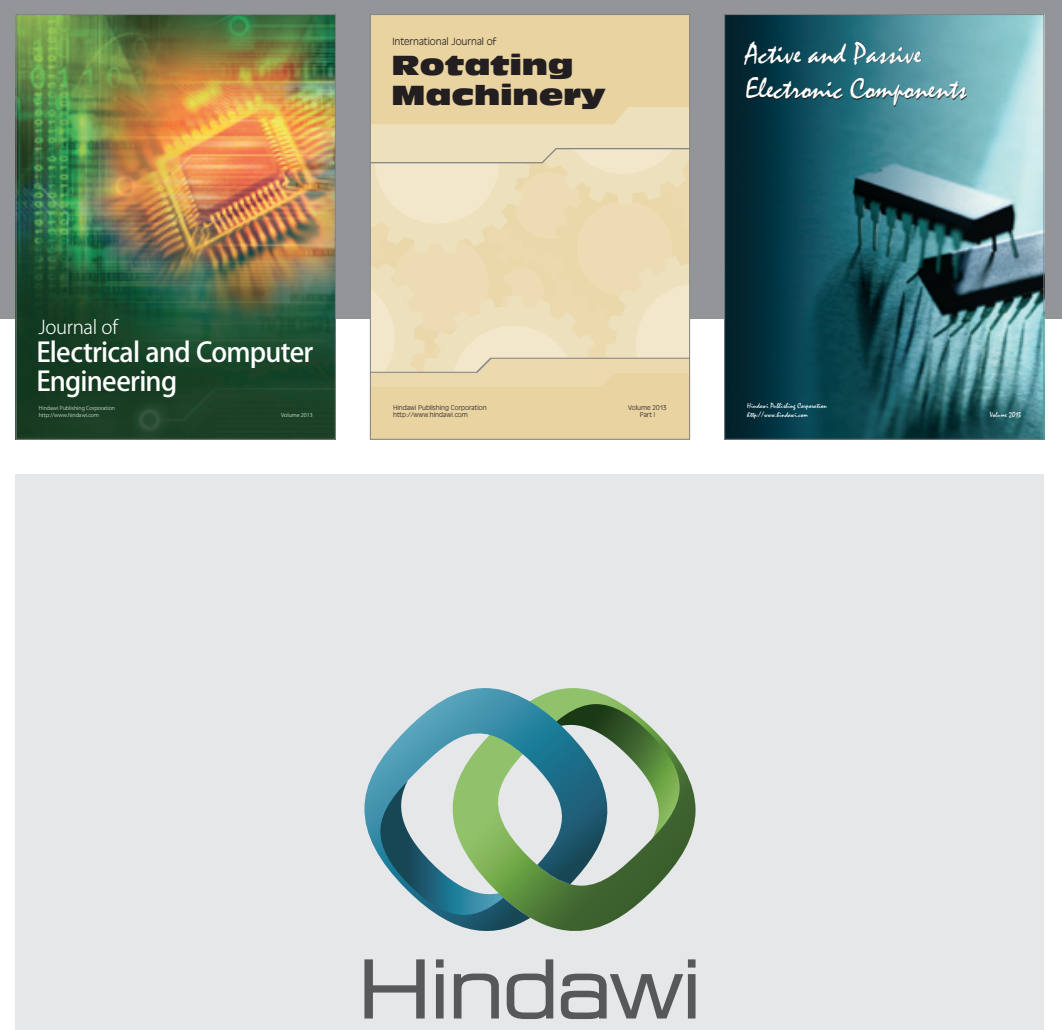

Submit your manuscripts at

http://www.hindawi.com
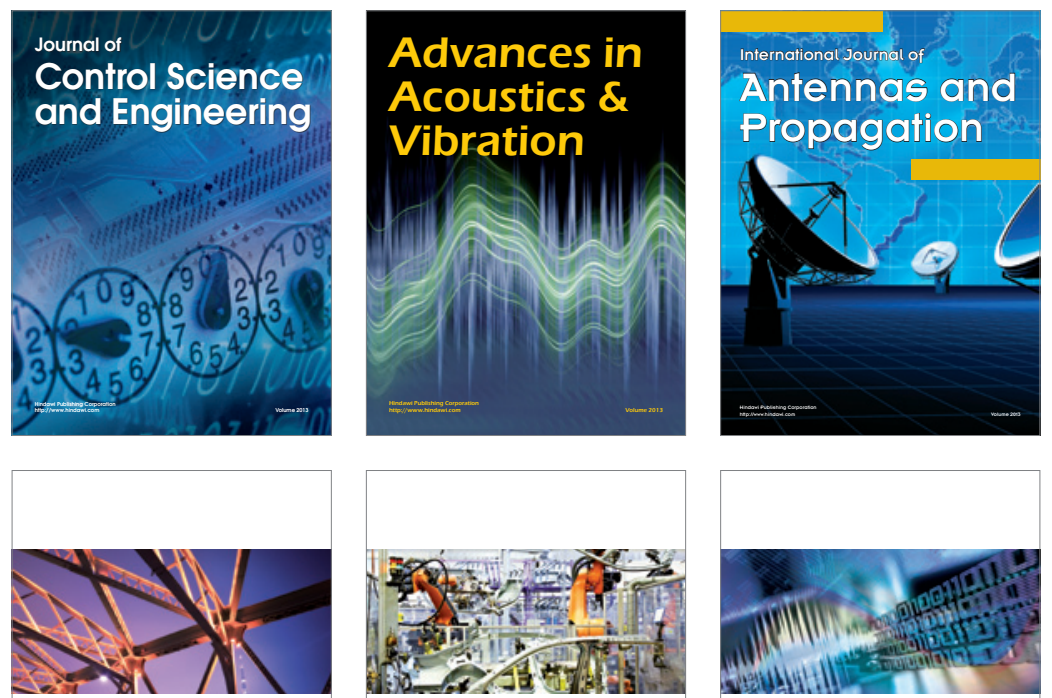

ISRN

Civil Engineering

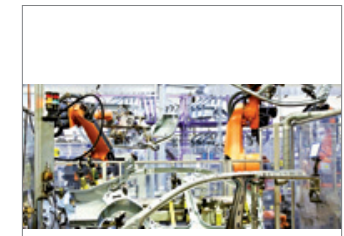

ISRN

Robotics

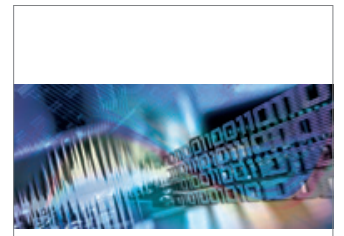

ISRN

Signal Processing
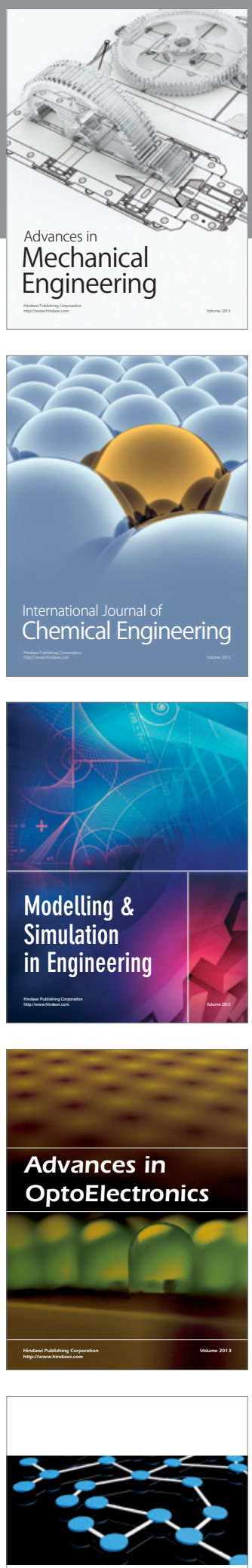

ISRN

Sensor Networks 